QUARTERLY OF APPLIED MATHEMATICS

VOLUME LXIX, NUMBER 2

JUNE 2011, PAGES 261-290

S 0033-569X(2011)01204-5

Article electronically published on March 3, 2011

\title{
TRANSIENT BEHAVIOR OF SOLUTIONS TO A CLASS OF NONLINEAR BOUNDARY VALUE PROBLEMS
}

\author{
BY \\ KURT BRYAN (Department of Mathematics, Rose-Hulman Institute of Technology, Terre Haute, \\ Indiana 47803) \\ AND \\ MICHAEL S. VOGELIUS (Department of Mathematics, Rutgers University, New Brunswick, New \\ Jersey 08903)
}

Abstract. In this paper we consider the asymptotic behavior in time of solutions to the heat equation with nonlinear Neumann boundary conditions of the form $\partial u / \partial \mathbf{n}=$ $F(u)$, where $F$ is a function that grows superlinearly. Solutions frequently exist for only a finite time before "blowing up." In particular, it is well known that solutions with initial data of one sign must blow up in finite time, but the situation for sign-changing initial data is less well understood. We examine in detail conditions under which solutions with sign-changing initial data (and certain symmetries) must blow up, and also conditions under which solutions actually decay to zero. We carry out this analysis in one space dimension for a rather general $F$, while in two space dimensions we confine our analysis to the unit disk and $F$ of a special form.

1. Introduction: the basic problem. Let $\Omega$ be a region in $\mathbb{R}^{N}$, with suitably smooth boundary, and let $u(x, t)$ be a solution to the heat equation

$$
\frac{\partial u}{\partial t}-\triangle u=0 x \in \Omega, 0<t<T,
$$

with the nonlinear Neumann boundary condition

$$
\frac{\partial u}{\partial \mathbf{n}}=F(u) \text { on } \partial \Omega
$$

( $\mathbf{n}$ is the outward unit normal vector) and initial condition

$$
u(x, 0)=f(x) x \in \Omega .
$$

Precise assumptions about the function $F$ will be stated later, but in general $F$ should be strictly increasing and grow superlinearly.

Received June 2, 2009.

1991 Mathematics Subject Classification. Primary 35B05, 35B40.

Key words and phrases. Blowup, heat equation, nonlinear Neumann boundary condition.

(C) 2011 Brown University 
Under these conditions, in particular, if the initial condition is positive, one may expect that the solution $u(x, t)$ will "blow up", that is, become unbounded, in finite time. Considerable work has been done on this problem to determine, for example, where in $\bar{\Omega}$ the function $u$ will blow up (in general, on some subset of $\partial \Omega$ ), to provide upper and lower bounds on the time at which blowup will occur, and to provide upper and lower bounds for the solution near blowup. Most of this work has been done for nonlinear functions $F$ of rather specific forms, typically $F(u)=u^{p}$ for $p>1$ or $F(u)=e^{u}$. Many variations of the above basic model have also been examined.

Early results on blowup for the heat equation with nonlinear Neumann boundary conditions were obtained in [20] and 31, where the authors demonstrate the inevitability of blowup for certain types of nonlinear boundary conditions and initial data, as well as for variations of the heat equation itself. Similar but more general versions of the problem are considered in [32]. In [12, the authors examine the problem on the unit ball for $F(u)=u^{p}$, and under certain conditions prove results concerning the blow-up rate and spatial profile of $u$ at the blow-up time. In [17, the authors consider a rather general case, a bounded domain $\Omega \subset \mathbb{R}^{N}, N \geq 2$, and show that blowup must occur for positive initial data. They establish upper and lower bounds for the quantity $\max _{x \in \bar{\Omega}} u(x, t)$ near blowup, for $F(u)=u^{p}$ with $1<p<(N-1) /(N-2)$ in the case $N>2$, and $1<p<\infty$ if $N=2$. They show that blowup occurs only on $\partial \Omega$ and make a detailed examination of the spatial profile of $u$ near blowup. Extensions of these results (to domains with less smooth boundaries, and to the case $N=2, F(u)=e^{u}$ ) are given in [16. Analogous results have been obtained for the case in which $\Omega$ is a half-space; see [7]. Extensions to coupled systems of two heat equations have been studied in [6, 21, 33, 34, for special domains and/or nonlinear boundary conditions. Results for slightly more general $F$ (but polynomially bounded) are given in [10. Extensions to the case in which reaction terms are present (e.g., $u_{t}=\Delta u+h(u)$ for certain $h$ ) or to the case of nonlinear diffusion are presented in [1, 26, 29, 30, 35. In some cases the emphasis is on proving and studying blowup; in others, the emphasis is on proving the global existence of solutions in time. Some papers focus on a careful analysis of the spatial set on which $u$ blows up (24]), and others (e.g., 11, 25]) consider the interesting question of whether solutions can be meaningfully continued past the blow-up time. In many cases the techniques used are similar to those used to study blowup in equations of the form $u_{t}-\Delta u=|u|^{p-1} u$ for $p>1$; see [13, 14, 15.

The articles 2, 9, 18, provide a more complete survey of results concerning the blowup of solutions to the heat equation with these types of nonlinear boundary conditions.

The present paper considers only space dimensions $N=1$ and $N=2$ and is devoted to the study of solutions with certain types of sign-changing initial data. In the first section of this paper, Section 2, we study criteria in the $N=1$ case for the initial condition $u(x, 0)=f(x)$ that guarantee either that $u(x, t)$ converges to zero as $t \rightarrow \infty$, or that $u(x, t)$ blows up in finite time. These criteria are related to solutions to the steady-state elliptic boundary value problem

$$
\Delta u=0 \text { in } \Omega, \frac{\partial u}{\partial \mathbf{n}}=F(u) \text { on } \partial \Omega
$$


and hold for rather general $F$; note that when $N=1$ this becomes the rather trivial equation $u^{\prime \prime}(x)=0$. The existence of nontrivial (nonconstant) solutions to the elliptic problem (44) does require that $F$ takes positive as well as negative values; in order to assure this we shall always require that $F$ be odd, i.e., $F(-u)=-F(u)$. In Section 3 we consider a very special two-space-dimensional case, namely $F(u)=\sinh (u)$ and $\Omega$ a disk, where a whole family of explicit solutions to the problem (4) is known.

To the best of our knowledge our results are the first to establish the role of nontrivial (explicit) steady-state solutions as pointwise barriers between blowup and decay for the time-dependent problem. The fact that these barriers apply to sign-changing solutions is also a novelty.

1.1. Assumptions. We start by making only very general assumptions about $F$, namely that

$$
F \in C^{2}(\mathbb{R}), F \text { is odd }(F(-u)=-F(u)), F^{\prime}(0)=1 \text {, and } F^{\prime \prime}(u)>0 \text { for } u>0 .
$$

Note that the last assumption implies that $F^{\prime}(u)$ is strictly increasing for $u>0$. The assumption that $F^{\prime}(0)=1$ is for convenience; we really need only that $F^{\prime}(0)>0$. By the odd symmetry, we have $F(0)=0$. We shall also require that $F$ grow superlinearly in the sense that

$$
u \frac{F^{\prime}(u)}{F(u)} \geq 1+\delta_{1}, \quad u \geq M
$$

for some positive constants $M$ and $\delta_{1}$. We note that

$$
\frac{d}{d u}\left(\frac{F(u)}{u^{1+\delta}}\right)=u^{\delta} F(u) \frac{u \frac{F^{\prime}(u)}{F(u)}-(1+\delta)}{u^{2+2 \delta}},
$$

and so as a consequence of (A2) (and the fact that $F$ is odd, with $F(u)>0, u>0$ ) it follows that

$$
\begin{aligned}
& \frac{F(u)}{|u|^{1+\delta}},|u| \geq M, \quad \text { is strictly increasing for any } \delta<\delta_{1}, \\
& \text { and nondecreasing for } \delta=\delta_{1} .
\end{aligned}
$$

The facts that $F(0)=0$ and $F^{\prime \prime}(u)>0$ for $u>0$ are easily seen to imply that $F(u)<$ $u F^{\prime}(u)$ when $u>0$, and therefore

$$
\frac{F(u)}{|u|} \text { is strictly increasing on all of } \mathbb{R} \text {. }
$$

From (5) and (6) it now follows that

$$
\frac{|F(u)|}{|u|^{1+\delta_{1}}} \geq C>0 .
$$

The facts that $F(0)=0$ and $F^{\prime}(0)=1$ ensure that $|F(u)| /|u|^{1+\delta_{1}}$ approaches infinity as $u \rightarrow 0$. We note that the strict monotonicity of $F^{\prime}(u)$ and $F(u) / u, u>0$, imply the existence of well-defined (positive-valued) inverses for these functions, say, for arguments larger than 1.

There are a variety of approaches for characterizing types of initial data $f$ that lead to blowup. For example, let $G$ be the anti-derivative for $F$ with $G(0)=0$; for quite general 
$F$ and "compatible" initial data $f$ it is possible to show that if the energy expression

$$
E(v)=\frac{1}{2} \int_{0}^{1}|\nabla v|^{2} d x-(G(v(1))+G(v(0)))
$$

is initially negative, that is, if $E(f)<0$ (or if $E(u)$ turns negative at any later time), then $u(x, t)$ will blow up in finite time, in the sense that the $H^{1}$ norm $\left(\int_{0}^{1}|\nabla u|^{2}(x, t) d x+\right.$ $\left.\int_{0}^{1} u^{2}(x, t) d x\right)^{1 / 2}$ will become infinite in finite time. The argument is a simple variation on the "concavity method" that dates back (at least) to the paper [19]. The requirement that $f$ be compatible with $F$ is needed in order to guarantee that $u$ is sufficiently smooth on $[0,1] \times[0, T]$ (and not just on $[0,1] \times(0, T]$ ). We won't make use of this onespace-dimensional blow-up criterion, but its analogue will be essential in our analysis of two-space-dimensional blowup. We examine this energy-based blow-up criterion more carefully in Appendix 4 .

2. Blowup vs. decay in one dimension. In one space dimension we may as well pick $\Omega=(0,1)$, and so the initial-boundary value problem becomes

$$
\begin{aligned}
\frac{\partial u}{\partial t}-\frac{\partial^{2} u}{\partial x^{2}} & =0,0<x<1,0<t<T, \\
-\frac{\partial u}{\partial x}(0, t) & =F(u(0, t)), 0<t<T, \\
\frac{\partial u}{\partial x}(1, t) & =F(u(1, t)), 0<t<T, \\
u(x, 0) & =f(x), 0<x<1 .
\end{aligned}
$$

Equations (8)-(11) can be reformulated as a pair of integral equations involving the functions $u_{0}(t):=u(0, t), u_{1}(t):=u(1, t)$ and the Green's function for the heat equation; see [5. If $f \in C^{0}([0,1])$ and $F$ is Lipschitz continuous, then a standard contraction mapping argument demonstrates the local existence of a solution to these integral equations and shows that the functions $u_{0}$ and $u_{1}$ are in $C^{\alpha}[0, T)$ for some $T>0$ and any $\alpha<1$.

In [5], we gave a simple argument to show that if the initial data $f$ is of one sign, then solutions must blow up. We also showed that blowup can only occur at the endpoints and we established precise lower and upper bonds on the blow-up rate. In the next section we complement this analysis by establishing a pointwise criteria on sign-changing initial data, with certain symmetries, that guarantees either blowup in finite time or global existence and decay to zero. The precise lower and upper bounds from [5] apply to these potential blow-up rates as well.

2.1. Decay and growth for certain symmetric solutions. We suppose that the initial condition $f$ is anti-symmetric about $x=1 / 2$, that is, $f(1-x)=-f(x)$. It is quite easy to show that the solution $u(x, t)$ inherits the same property, $u(1-x, t)=-u(x, t)$, for all $t>0$ in the domain of existence; in particular, $u(1 / 2, t)=0$. In this section we shall analyze criteria that make it possible to predict whether solutions decay to zero uniformly over the domain $0 \leq x \leq 1$ as $t \rightarrow \infty$, or blow up in finite time at the endpoints.

REMARK 1. It should be clear that the behavior of $F(x)$ for $x$ sufficiently near zero has no bearing on solutions that blow up, and conversely, the behavior of $F(x)$ for $x$ sufficiently large has no bearing on solutions that decay. Thus, for example, the condition 
$F(0)=0$ is not relevant to the blow-up analysis that follows, and the growth bounds stemming from (A2) are not relevant for the decay results.

Many of the arguments in this section are straightforward comparison principles that rely on the maximum principle. Let us thus first establish the following lemma.

Lemma 2.1. Let $\Omega=(a, b)$ be a bounded interval. Let $u(x, t)$ and $v(x, t)$ be solutions to the heat equation in $\Omega \times(0, T)$, each in $C^{1}\left((0, T) ; C^{2}(\Omega)\right) \cap C^{0}\left((0, T) ; C^{1}(\bar{\Omega})\right) \cap$ $C^{0}\left([0, T) ; C^{0}(\bar{\Omega})\right)$, with $u=v=0$ at $x=a$ and Neumann data

$$
\frac{\partial u}{\partial x}=F(u), \quad \frac{\partial v}{\partial x}=G(v)
$$

at the right endpoint $x=b$. Let $Z=\{z$ : there exists $t$ with $0<t<T$ such that $z=$ $u(b, t)=v(b, t)\}$ and suppose that $F(z) \leq G(z)$ for all $z \in Z$. Suppose also that $u$ and $v$ have initial data $u(x, 0)=f(x), v(x, 0)=g(x)$, with $f(x)<g(x)$ for $x \in(a, b]$. Then $u(x, t)<v(x, t)$ for all $x \in(a, b]$ and $t \in[0, T)$.

Proof. Let $w=v-u$, so that $w$ has the same regularity as $u$ and $v$ and also satisfies the heat equation. We have $w=0$ at $x=a$ and $w(x, 0)>0$ for $x \in(a, b]$. Let $0<T^{\prime}<T$. According to the maximum principle (or in this case, the minimum principle) the minimum value for $w$ on $[a, b] \times\left[0, T^{\prime}\right]$ is assumed either at $t=0$ or at $x=a$ or $x=b$ with $0<t \leq T^{\prime}$. In fact, the minimum cannot be attained at any point $\left(x_{1}, t_{1}\right)$ in $(a, b) \times\left(0, T^{\prime}\right]$ unless $w$ is constant for $t \leq t_{1}$ (see, e.g., Theorem 2 of Section 2 , Chapter 3 in [23]); our assumptions on the initial data preclude $w$ being constant on any such set. Indeed, the assumptions show that the minimum here must occur at $x=a$ (where $w \equiv 0$ ) or $x=b$.

We now show that $w(b, t)>0$ for all $0 \leq t \leq T^{\prime}$. Suppose to the contrary that

$$
\left\{0 \leq t \leq T^{\prime}: w(b, t) \leq 0\right\} \neq \emptyset,
$$

and let $t^{*}$ denote the infimum of this set. From the assumptions we clearly have $t^{*}>0$, and continuity yields $w\left(b, t^{*}\right)=0$. Thus $w$ attains its minimum value on $[a, b] \times\left[0, t^{*}\right]$ at the point $(x, t)=\left(b, t^{*}\right)$ (as well as anywhere on $x=a$ ). From Theorem 3 of Section 2, Chapter 3 in 23 we conclude that, since $w$ is not constant,

$$
\frac{\partial w}{\partial x}\left(b, t^{*}\right)<0 .
$$

But from the assumptions in the statement of this lemma we have

$$
\begin{aligned}
\frac{\partial w}{\partial x}\left(b, t^{*}\right) & =\frac{\partial v}{\partial x}\left(b, t^{*}\right)-\frac{\partial u}{\partial x}\left(b, t^{*}\right) \\
& =G\left(v\left(b, t^{*}\right)\right)-F\left(u\left(b, t^{*}\right)\right) \\
& \geq 0 \text { since } v\left(b, t^{*}\right)=u\left(b, t^{*}\right),
\end{aligned}
$$

in contradiction to inequality (12). We conclude that the set $\left\{0 \leq t \leq T^{\prime}: w(b, t) \leq 0\right\}$ is empty, and so $w(b, t)>0$ for all $t \in\left[0, T^{\prime}\right]$. That $w(x, t)>0$ for $x \in(a, b)$ and $t \in\left[0, T^{\prime}\right]$ follows from the fact that the minimum value assumed by $w$ on $[a, b] \times\left[0, T^{\prime}\right]$ is 0 and, as remarked above, $w(x, t)$ cannot assume this minimum value for $a<x<b$, $0 \leq t \leq T$. In summary we have proven that $w(x, t)>0$ and thus that $u(x, t)<v(x, t)$ 
for $a<x \leq b, 0 \leq t \leq T^{\prime}$. Since $T^{\prime}$ is an arbitrary time smaller than $T$, this completes the proof of Lemma 2.1

We now proceed with an analysis of the steady-state case for (8)-(11). This is straightforward, for then $u=u(x)$ satisfies $u^{\prime \prime}(x)=0$, and hence (due to the symmetries assumed) is of the form $u(x)=m(x-1 / 2)$. The boundary conditions yield the requirement that $m=F(m / 2)$. From the conditions imposed on $F$ (in particular that $F^{\prime}(0)=1$ and that $F(x)$ is of superlinear growth) it follows that the equation $m=F(m / 2)$ has precisely three roots, $m=0, m=m_{*}>0$, and $m=-m_{*}$, and corresponding solutions $u \equiv 0$, $u(x)=m_{*}(x-1 / 2)$, and $u(x)=-m_{*}(x-1 / 2)$. We note that for three steady-state solutions to emerge it is not essential that $F^{\prime}(0)=1$, rather the condition $0<F^{\prime}(0)<2$ would suffice.

The steady-state solutions can be used in a comparison argument to predict decay or finite time blowup of the transient solutions. We start by establishing the following boundedness lemma.

Lemma 2.2. Let $m_{*}$ be the positive solution to $m_{*}=F\left(m_{*} / 2\right)$, and let $u(x, t)$ be the solution to equations (8) -(11) with initial condition $f(x) \in C^{0}([0,1])$ that has the odd symmetry $f(1-x)=-f(x)$, and satisfies $|f(x)| \leq m|x-1 / 2|, x \in[0,1]$, for some $m \in\left[0, m_{*}\right)$. Then $u(x, t)$ can be continued as a solution for all $t>0$, and $|u(x, t)| \leq m|x-1 / 2|$ for $x \in[0,1]$ and all $t>0$.

Proof. As already remarked, the solution $u(x, t)$ exists for some time interval $\left(0, t_{0}\right)$. We will establish the inequality $|u(x, t)| \leq m|x-1 / 2|$ on any such interval, so that the same contraction mapping argument that established the local existence may be used to extend $u$ beyond $t_{0}$; this will imply that $u$ is defined for all $t>0$ and satisfies $|u(x, t)| \leq m|x-1 / 2|$.

Given the symmetry of $f$ and $u$, we restrict our attention to the interval $x \in[1 / 2,1]$. Let us first focus on the inequality $f(x) \leq m(x-1 / 2)$. Choose $m^{\prime} \in\left(m, m^{*}\right)$ and define $v(x, t)=m^{\prime}(x-1 / 2)$. The function $v$ satisfies the heat equation with $v(1 / 2, t)=0$ and

$$
v_{x}(1, t)=G(v(1, t))
$$

where $\left(\right.$ since $\left.v_{x}(1, t)=m^{\prime}, v(1, t)=m^{\prime} / 2\right)$ we have $G(z)=2 z$. Note that $F(z) \leq 2 z=$ $G(z)$ for $z \in\left[0, m_{*} / 2\right]$ since by ([6),$F(z) / z$ is increasing, and $F\left(m_{*} / 2\right) / m_{*}=1$.

Due to the fact that $m<m^{\prime}$ it now follows from Lemma 2.1 applied to the solutions $u$ and $v$ that $u(x, t)<v(x, t)=m^{\prime}(x-1 / 2)$ for all $0 \leq t<t_{0}, 1 / 2<x \leq 1$. By letting $m^{\prime}$ tend to $m$ we may conclude that $u(x, t) \leq m(x-1 / 2)$ for $x \in[1 / 2,1]$. A similar argument may be made for the inequality $u(x, t) \geq-m(x-1 / 2)$ for $x \in[1 / 2,1]$ and all $t<t_{0}$, and this completes the proof of Lemma 2.2

REMARK 2. We note that if $m>m_{*}$ and the odd symmetric initial condition satisfies $f(x) \geq m|x-1 / 2|$ for all $x \in[1 / 2,1]$, or $f(x) \leq-m|x-1 / 2|$ for all $x \in[1 / 2,1]$, then an argument almost identical to that of the proof of Lemma 2.2 shows that $u(x, t) \geq$ $m|x-1 / 2|$ or $u(x, t) \leq-m|x-1 / 2|$, respectively, for all $x \in[1 / 2,1]$, and those $t$ for which the solution exists. 
Proposition 2.3. Under the assumptions (A1), (A2), and those of Lemma 2.2 we have

$$
\lim _{t \rightarrow \infty} u(x, t)=0
$$

uniformly with respect to $x \in[0,1]$.

Proof. As before we may consider only $x \in[1 / 2,1]$. Let $m^{\prime} \in\left(m, m_{*}\right)$. From Lemma 2.2 we have $|u(x, t)| \leq m|x-1 / 2|$ for all $t$, and in particular $|u(1, t)| \leq m / 2$. We also note that

$$
\frac{F(z)}{z} \leq \frac{F(m / 2)}{m / 2}<B_{0}:=\frac{F\left(m^{\prime} / 2\right)}{m^{\prime} / 2}<2 \text {, for all } 0 \leq z \leq m / 2 .
$$

Let $v(x, t)$ denote the solution to the heat equation on $1 / 2<x<1$ with boundary conditions $v(1 / 2, t)=0$, Neumann data $v_{x}(1, t)=B_{0} v(1, t)$, and initial data $v(x, 0)=$ $m^{\prime}(x-1 / 2)$. The maximum principle guarantees that $v(1, t)>0$ for all $t \geq 0$.

The hypotheses of Lemma 2.1 are met with $G(z)=B_{0} z$ (since common values of $u(1, t)$ and $v(1, t)$ lie between 0 and $m / 2)$. We conclude that $u(x, t)<v(x, t)$ for all $x \in(1 / 2,1]$ and $t \geq 0$. A similar argument establishes that $u(x, t)>-v(x, t)$, so that $|u(x, t)| \leq v(x, t)$ for all $x \in[1 / 2,1]$ and $t \geq 0$.

We can solve for $v$ explicitly with a straightforward separation of variables to find that

$$
v(x, t)=\sum_{k=1}^{\infty} A_{k} e^{-q_{k}^{2} t} \sin \left(q_{k}(x-1 / 2)\right),
$$

where the $q_{1}<q_{2}<\ldots$ are the countably many positive roots of

$$
q=B_{0} \tan (q / 2),
$$

and the sequence $\left\{A_{k}\right\}_{k=1}^{\infty} \in \ell^{2}$ can be determined from the initial condition. The root $q_{1}$ lies in the interval $(0, \pi)$, and the $q_{k}$ are asymptotically equal to $(2 k-1) \pi$ as $k \rightarrow \infty$. Therefore

$$
\begin{aligned}
|v(x, t)| \leq \sum_{k}\left|A_{k}\right| e^{-q_{k}^{2} t} & \leq e^{-q_{1}^{2} t} \sum_{k}\left|A_{k}\right| e^{-\left(q_{k}^{2}-q_{1}^{2}\right) t} \\
& \leq M_{0} e^{-q_{1}^{2} t} \text { for } t>t_{0}>0, x \in[1 / 2,1],
\end{aligned}
$$

In other words, $|v(x, t)|$ converges to 0 as $t \rightarrow \infty$, uniformly with respect to $x$. This completes the proof of Proposition 2.3 .

REMARK 3 . If the initial condition $f$ has an odd symmetry about $x=1 / 2$ and satisfies $f(x) \geq m|x-1 / 2|$ for $x \in[1 / 2,1]$, or $f(x) \leq-m|x-1 / 2|$ for $x \in[1 / 2,1], m>m_{*}$, then an argument similar to that in the proof of the last lemma can be used to examine the growth of $u$. Briefly, select $m^{\prime}$ so that $m_{*}<m^{\prime}<m$ and define $B_{0}:=\frac{F\left(m^{\prime} / 2\right)}{m^{\prime} / 2}>\frac{F\left(m_{*} / 2\right)}{m_{*} / 2}=2$. The argument of Proposition 2.3 shows that $|u(x, t)| \geq|v(x, t)|$, where $v$ is the solution to the heat equation on $1 / 2<x<1$ with $v(1 / 2, t)=0, v_{x}(1, t)=B_{0} v(1, t)$, and initial condition $v(x, 0)=m^{\prime}(x-1 / 2)$. A straightforward separation of variables yields

$$
v(x, t)=A_{1} e^{q_{1}^{2} t} \sinh \left(q_{1}(x-1 / 2)\right)+\sum_{k=2}^{\infty} A_{k} e^{-q_{k}^{2} t} \sin \left(q_{k}(x-1 / 2)\right),
$$

where the $q_{k}$, for $k \geq 2$, are the countably many positive roots of $q=B_{0} \tan (q / 2)$ and $q_{1}$ is the unique positive root of $q=B_{0} \tanh (q / 2)$, the existence of which is assured by the 
fact that $B_{0}>2$. Since $\tanh (q / 2)<1$ it follows immediately that $q_{1}<B_{0}$. Moreover, because

$$
\frac{q / 2}{\tanh (q / 2)}<\frac{q}{2}+1, q>0
$$

it follows immediately that

$$
B_{0} / 2<\frac{q_{1}}{2}+1, \text { or } B_{0}-2<q_{1} .
$$

In summary, $0<B_{0}-2<q_{1}<B_{0}$. The value of $A_{1}$ in (13) is determined as a ratio of $L^{2}(1 / 2,1)$ inner products, namely $A_{1}=\left\langle m^{\prime}(x-1 / 2), \sinh \left(q_{1}(x-1 / 2)\right)\right\rangle /\left\langle\left(\sinh \left(q_{1}(x-\right.\right.\right.$ $\left.1 / 2)), \sinh \left(q_{1}(x-1 / 2)\right)\right\rangle$, and is clearly positive. As a consequence $v$ grows without bound as $t \rightarrow \infty$. This would also immediately prove that $u$ grows without bound if $u$ were defined for all $t$. However, we can do better: in the following we shall prove that $u$ becomes unbounded in finite time.

2.2. Finite time blowup for certain solutions. Before stating the finite time blow-up result, we need to introduce what will amount to an upper bound on the time of existence. Given any $m>m_{*}$ we define $B_{k}(m)$ as

$$
B_{k}(m):=\frac{F\left(m 2^{k-1}\right)}{m 2^{k-1}}, k=0,1, \ldots .
$$

Due to the assumptions about $F$ (that lead to (6) -(77) ) and the definition of $m_{*}$ as the positive root of $z=F(z / 2)$ we have

$$
2<B_{0}(m)<B_{1}(m)<B_{2}(m)<\ldots, \text { with } B_{k}(m) \geq c_{m}\left(2^{\delta_{1}}\right)^{k},
$$

for some constant $c_{m}>0$, independent of $k$. The constant $B_{0}$ used in the proof of Proposition 2.3 and Remark 3 coincides with $B_{0}\left(m^{\prime}\right)$ according to this definition. From the assumptions about $F$ it follows that $B_{k}\left(m^{\prime}\right)<B_{k}(m)$ for $m_{*}<m^{\prime}<m$, and that $\lim _{m^{\prime} \rightarrow m} B_{k}\left(m^{\prime}\right)=B_{k}(m)$. For any $m>m_{*}$ we now define

$$
T(m)=\sum_{k=0}^{\infty}\left(B_{k}(m)-2\right)^{-2}+\sum_{k=0}^{\infty}\left(B_{k}(m)-2\right)^{-1} \text {. }
$$

The fact that $T(m)<\infty$ for any $m>m_{*}$ follows immediately from (15). From the monotonicity and convergence properties of the $B_{k}$ it follows that $\lim _{m^{\prime} \rightarrow m} T\left(m^{\prime}\right)=$ $T(m)$. Since $B_{0}(m) \rightarrow 2$ as $m \rightarrow m_{*}$ we also see that $T(m) \rightarrow \infty$ as $m \rightarrow m_{*}$.

Proposition 2.4. Let $m_{*}$ be the unique positive solution to $m_{*}=F\left(m_{*} / 2\right)$, where $F$ satisfies (A1) and (A2), and let $m>m_{*}$. Let $u(x, t)$ be a solution to equations (8)-(11) with initial condition $f \in C^{0}([0,1])$ that has the odd symmetry $f(1-x)=-f(x)$ about $x=1 / 2$, and satisfies $|f(x)| \geq m|x-1 / 2|$ for all $x \in[1 / 2,1]$. Let $T(m)$ be as defined in (16). Then there exists $t^{*} \leq T(m)$ such that

$$
\begin{aligned}
& \text { (1) } u(1, \cdot) \text { is in } C^{\alpha}\left[0, t^{*}\right) \text { for any } \alpha<1, \\
& \text { (2) } u(1, \cdot) \text { fails to be bounded as } t \text { approaches } t^{*} \text {. }
\end{aligned}
$$

Proof. As remarked shortly after equations (8)-(11), for any $\alpha<1$ the function $u(1, t)$ will be in $C^{\alpha}\left[0, t_{1}\right)$ on any interval $0 \leq t<t_{1}$ on which $u(1, t)$ remains bounded. For any such $\alpha$ define

$$
T=\sup \left\{t>0: u(1, \cdot) \in C^{\alpha}[0, t) \cap L^{\infty}(0, t)\right\}
$$


If $T$ is finite, then $u(1, t)$ will be in $C^{\alpha}[0, T)$ but will fail to be in $L^{\infty}(0, T)$ (because if not, then a contraction mapping argument would prove smooth existence beyond $T$ ). There are two possibilities: (1) $T \leq T(m)$, or $(2) T>T(m)$. In the first scenario we know that $u(1, \cdot)$ is in $C^{\alpha}[0, t)$ for any $t<T$ but not in $L^{\infty}(0, T)$. This partially verifies the proposition with $t^{*}=T \leq T(m)$. We now proceed to show that the second scenario cannot occur, thus completing the proof of the proposition.

We will focus on the interval $1 / 2 \leq x \leq 1$ and the case $u(x, 0) \geq m(x-1 / 2)$; the alternative case $u(x, 0) \leq-m(x-1 / 2)$ can be treated in an entirely similar fashion. Now suppose that $T>T(m)$; since $\lim _{m^{\prime} \rightarrow m} T\left(m^{\prime}\right)=T(m)$ we have that $T>T\left(m^{\prime}\right)$ for all $m_{*}<m^{\prime}<m$ with $m^{\prime}$ sufficiently close to $m$. The essential idea in the argument that follows is to estimate the time $t_{1}$ that it takes a solution $u(x, t)$ with initial condition $u(x, 0) \geq m(x-1 / 2)$ to grow until $u\left(x, t_{1}\right) \geq 2 m(x-1 / 2)$ is satisfied. Recall from Remark 2 that we already have that $u(x, t) \geq m(x-1 / 2)$ for all $t$ for which the solution $u$ exists.

As before let $q_{1}=q_{1}\left(m^{\prime}\right)$ be the positive root of $q=B_{0}\left(m^{\prime}\right) \tanh (q / 2)$ and define

$$
v(x, t)=\frac{m^{\prime}}{2 \sinh \left(q_{1} / 2\right)} e^{q_{1}^{2} t} \sinh \left(q_{1}(x-1 / 2)\right) .
$$

This function satisfies the heat equation with $v(1 / 2, t)=0$ and $v_{x}(1, t)=B_{0}\left(m^{\prime}\right) v(1, t)$. Moreover, $v(1,0)=m^{\prime} / 2$, and since $v(x, 0), x \geq 1 / 2$, is convex (with $v(1 / 2,0)=0$ ) we have $v(x, 0) \leq m^{\prime}(x-1 / 2)$ for $x \in[1 / 2,1]$. A comparison argument similar to that already given in Lemma 2.2 shows that $v(x, t) \leq u(x, t)$ for all $x \in[1 / 2,1]$ and $0 \leq t<T$. A simple computation shows that $v_{x}(1 / 2, t)=\frac{m^{\prime} q_{1} e^{q_{1}^{2} t}}{2 \sinh \left(q_{1} / 2\right)}$, and since $v(x, t)$ is convex for $x \geq 1 / 2$ we can bound

$$
\frac{m^{\prime} q_{1} e^{q_{1}^{2} t}}{2 \sinh \left(q_{1} / 2\right)}(x-1 / 2) \leq v(x, t) \leq u(x, t), 1 / 2 \leq x \leq 1,0 \leq t<T .
$$

We introduce

$$
t_{1}=t_{1}\left(m^{\prime}\right)=\frac{1}{q_{1}^{2}\left(m^{\prime}\right)} \ln \left[4 \sinh \left(q_{1}\left(m^{\prime}\right) / 2\right) / q_{1}\left(m^{\prime}\right)\right] .
$$

A straightforward estimation shows that

$$
t_{1} \leq \frac{\ln 2}{q_{1}^{2}}+\frac{1}{2 q_{1}} \leq q_{1}^{-2}+q_{1}^{-1}
$$

and in combination with (14) we find

$$
t_{1}\left(m^{\prime}\right) \leq\left(B_{0}\left(m^{\prime}\right)-2\right)^{-2}+\left(B_{0}\left(m^{\prime}\right)-2\right)^{-1}<T\left(m^{\prime}\right) .
$$

Insertion of $t_{1}\left(m^{\prime}\right)$ into (18) gives

$$
2 m^{\prime}(x-1 / 2) \leq u\left(x, t_{1}\left(m^{\prime}\right)\right) .
$$

Passage to the limit $m^{\prime} \rightarrow m$ in (19), (20) now yields

$2 m(x-1 / 2) \leq u\left(x, t_{1}(m)\right) \quad$ and $\quad t_{1}(m) \leq\left(B_{0}(m)-2\right)^{-2}+\left(B_{0}(m)-2\right)^{-1}<T(m)$.

We define $t_{2}$ in the same way as $t_{1}$, but with $m$ replaced by $2 m$, and so obtain

$$
t_{2}(m) \leq\left(B_{0}(2 m)-2\right)^{-2}+\left(B_{0}(2 m)-2\right)^{-1}=\left(B_{1}(m)-2\right)^{-2}+\left(B_{1}(m)-2\right)^{-1} .
$$


Therefore

$$
t_{1}+t_{2} \leq \sum_{k=0}^{1}\left[\left(B_{k}(m)-2\right)^{-2}+\left(B_{k}(m)-2\right)^{-1}\right]<T(m) .
$$

We now "start" the heat equation at $t=t_{1}(m)$ with initial datum $u\left(x, t_{1}(m)\right)$ and then advance time by $t_{2}(m)$ to get

$$
4 m(x-1 / 2) \leq u\left(x, t_{1}(m)+t_{2}(m)\right) .
$$

This process may be continued iteratively, yielding

$$
\sum_{k=1}^{K+1} t_{k}(m) \leq \sum_{k=0}^{K}\left[\left(B_{k}(m)-2\right)^{-2}+\left(B_{k}(m)-2\right)^{-1}\right]<T(m)
$$

and

$$
2^{K+1} m(x-1 / 2) \leq u\left(x, \sum_{k=1}^{K+1} t_{k}(m)\right),
$$

for any $K \geq 1$. At $x=1$ this forces

$$
2^{K} m \leq u\left(1, \sum_{k=1}^{K+1} t_{k}(m)\right), \text { where } \sum_{k=1}^{K+1} t_{k}(m) \leq T(m)<T,
$$

for any $K \geq 1$, in contradiction to the fact that $u(1, \cdot) \in L^{\infty}(0, T(m))$. We conclude that the second scenario $(T>T(m))$ cannot arise, and this completes the proof of Proposition 2.4 .

2.3. Examples. In Figure 1 we show the solution $u(x, t)$ corresponding to $F(u)=$ $u+u^{3}$. The numerical solution was obtained using a simple adaptive time-marching scheme based on an integral equation formulation of the boundary value problem (see, e.g., [5]). The nontrivial steady-state solutions are $\phi(x)= \pm 2(x-1 / 2)\left(\right.$ here $\left.m_{*}=2\right)$. The initial condition is $u(x, 0)=2 \tanh (x-1 / 2)$, shown as the solid curve; note that $u(x, 0)$ is odd in $x=1 / 2$, and satisfies $u(x, 0)<2|x-1 / 2|$ for $1 / 2<x \leq 1$, though we don't quite have $u(x, 0) \leq m|x-1 / 2|$ for any $m<2$ (as required by Proposition 2.3). Nonetheless, we see that the solution still decays to zero as $t \rightarrow \infty$. The solution $u(x, t)$ is shown at times $t=0.15$ and $t=0.5$.

In Figure 2 we again show the solution $u(x, t)$ corresponding to $F(u)=u+u^{3}$, but with initial condition $u(x, 0)=2.2 \tanh (x-1 / 2)$ (so $u(x, 0) \geq m(x-1 / 2)$ with $m=2.03>m_{*}=2$ ), shown as the solid curve. As predicted by Proposition 2.4, the solution blows up in finite time. The solution $u(x, t)$ is shown at times $t=0.1$ and $t=0.1232$, at which point the solution has "blown up," to numerical precision. Equation (16) yields $T(2.2) \approx 27.84$, so the upper bound in this case is not terribly sharp.

3. A two-space-dimensional case. An analysis similar to that in the previous section can be carried out in two space dimensions, though in this case one typically doesn't have the luxury of closed-form solutions to the elliptic nonlinear boundary value problem, analogous to $\phi(x)=m_{*}(x-1 / 2)$ on the interval $(0,1)$. However, if the domain $\Omega$ is the two-dimensional unit disk $\left(B_{1}\right)$, and if $F$ is of a special form, then a countable family of nontrivial, explicit solutions has been obtained, and these may be used as a basis for the analysis. 


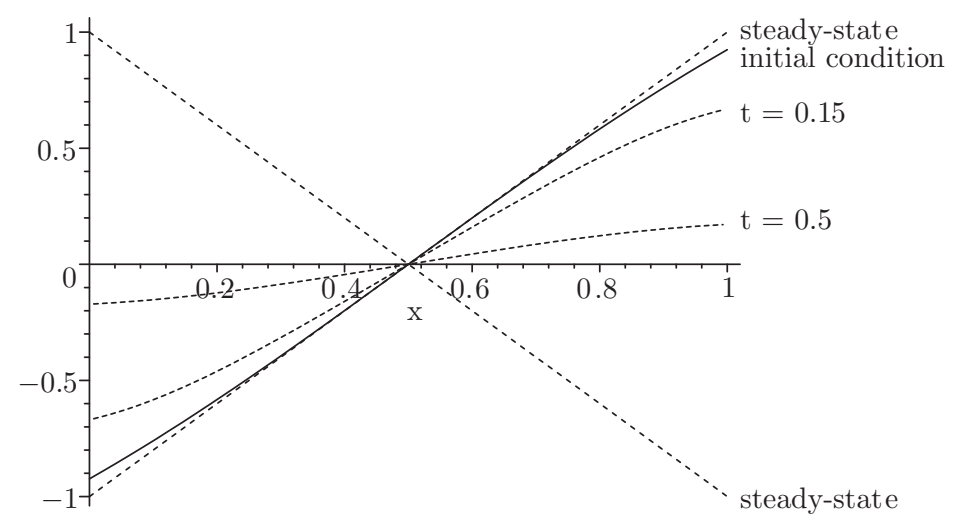

FIG. 1. $F(u)=u+u^{3}$, steady-state solution, initial condition, and $u(x, t)$ at $t=0.15,0.5$.

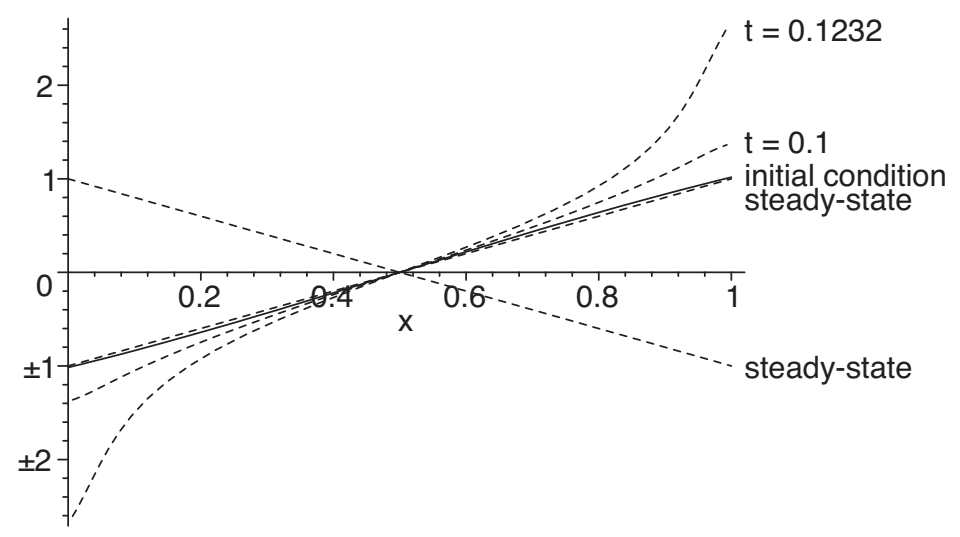

Fig. 2. $F(u)=u+u^{3}$, steady-state solutions, initial condition, and $u(x, t)$ at $t=0.1$ and $t=0.1232$.

In [4], the authors considered the elliptic boundary value problem $\triangle \phi=0$ on the unit disk $B_{1}$ with the boundary condition $\frac{\partial \phi}{\partial \mathbf{n}}=\lambda F(\phi)$ on $\partial B_{1}$, where

$$
F(z)=\sinh (z) 1
$$

and $\lambda>0$. This $F$ satisfies all the superlinearity conditions stated in Section 1.1; furthermore it is easy to see that for any given $\gamma<1$ we have

$$
\frac{|F(z)|}{|z|} \geq c e^{\gamma|z|}, z \neq 0
$$

for some $c>0$. The derivative of the flux at 0 is not 1 ; instead $(\lambda F)^{\prime}(0)=\lambda$. The parameter $\lambda$ is assumed to be positive, for otherwise the nonlinear elliptic boundary value problem has only the trivial solution $u \equiv 0$. For any fixed $\lambda>0$ a countable family

\footnotetext{
${ }^{1}$ The function considered in 4 is actually $2 \sinh (z / 2)$, but the change of dependent variable $u / 2 \rightarrow u$ gives rise to the $F$ introduced above.
} 
of closed-form solutions is given by

$$
\phi_{n}(x, y)=\sum_{k=0}^{2 n-1}(-1)^{k} K\left((x, y)-\mu(\lambda) p_{k}\right), n=1,2, \ldots,
$$

where $p_{k}:=\left(x_{k}, y_{k}\right)=(\cos (k \pi / n), \sin (k \pi / n)), 0 \leq k \leq 2 n-1$, are $2 n$ equispaced points on $\partial B_{1}, K(x, y)=\ln \left(x^{2}+y^{2}\right)$ (just $4 \pi$ times the usual Green's function for the Laplacian), and

$$
\mu(\lambda)=\left(\frac{n+\lambda}{n-\lambda}\right)^{\frac{1}{2 n}} .
$$

The solution $\phi_{n}$ is well-defined for $\lambda \in(0, n)$. Note that $\phi_{n}$ depends on $\lambda$, but we do not explicitly indicate this. As $\lambda \rightarrow 0^{+}$we find $\mu(\lambda) \rightarrow 1^{+}$and the function $\phi_{n}(x, y)$ develops logarithmic singularities at the $2 n$ boundary points $\left(x_{k}, y_{k}\right), 0 \leq k \leq 2 n-1$. The function $\phi_{n}(x, y)$ has some obvious symmetries:

$$
\begin{gathered}
\phi_{n}(x, y) \text { is evenly symmetric about any line } L_{k} \text { through the origin at angle } \\
\qquad \theta=\frac{k}{n} \pi, k=0,1, \ldots, 2 n-1, \text { with respect to the } x \text {-axis }
\end{gathered}
$$

and

$$
\begin{aligned}
& \phi_{n}(x, y) \text { is oddly symmetric about any line } L_{k}^{\prime} \text { through the origin at angle } \\
& \quad \theta=\frac{2 k+1}{2 n} \pi, k=0,1, \ldots, 2 n-1 \text {, with respect to the } x \text {-axis } .
\end{aligned}
$$

We suspect that the $\phi_{n}$ 's, modulo rotations, represent all solutions to the nonlinear boundary value problem with Neumann data $\lambda \sinh (u)$ on $B_{1}$. In what follows we will consider solutions $u(x, y, t)$ to

$$
\begin{aligned}
u_{t}-\triangle u & =0 \text { in } B_{1} \times(0, T), \\
\frac{\partial u}{\partial \mathbf{n}} & =\lambda F(u) \\
& =\lambda \sinh (u) \text { on } \partial B_{1} \times(0, T), \\
u(x, y, 0) & =f(x, y) \text { in } B_{1},
\end{aligned}
$$

where $\lambda>0$. We will assume that the initial condition, $f$, is a continuously differentiable function that possesses the same symmetry properties (24) and (25) as $\phi_{n}$, and that $f$ is "compatible" with the nonlinear boundary condition in the sense that $\frac{\partial f}{\partial \mathbf{n}}=\lambda F(f)=$ $\lambda \sinh (f)$ on $\partial B_{1}$. It is well known that a unique strong solution $u(x, y, t)$ exists for some interval of time. As a consequence of uniqueness, the solution $u$ also possesses the spatial symmetries (24) and (25). Furthermore, based on standard regularity theory (or an integral representation formula) it should be possible to show that this strong solution $u(x, y, t), 0<t<T$, is indeed an element of $C^{1}\left((0, T) ; \mathfrak{A}\left(\overline{B_{1}}\right)\right.$ ) (with $\mathfrak{A}\left(\overline{B_{1}}\right)$ denoting the real analytic functions in the spatial variables $\left.(x, y) \in \overline{B_{1}}\right)$. In light of this, for the purpose of the present analysis, we define a classical solution to (26)-(28) as a solution with

$$
u(x, y, t) \in C^{0}\left([0, T) ; C^{1}\left(\overline{B_{1}}\right)\right) \cap C^{1}\left((0, T) ; \mathfrak{A}\left(\overline{B_{1}}\right)\right) .
$$


3.1. Boundedness of certain solutions. We now prove two-dimensional analogues of the results from Section 2, in which each $\phi_{n}$ plays the role of the linear steady-state solution. We begin with a two-dimensional analogue of Lemma 2.2 .

Lemma 3.1. Let $\phi_{n}$ denote the steady-state solution defined by (23). Let $u(x, y, t), 0 \leq$ $t<T$, be a classical solution to the diffusion problem (26)-(28), with initial condition $f$ satisfying the same symmetry conditions (24)-(25) as $\phi_{n}$, and the compatibility condition $\frac{\partial f}{\partial \mathbf{n}}=\lambda F(f)=\lambda \sinh (f)$ on $\partial B_{1}$. If the initial condition additionally satisfies $|f(x, y)| \leq$ $m\left|\phi_{n}(x, y)\right|,(x, y) \in B_{1}$, for some $m \in(0,1)$, then $|u(x, y, t)| \leq m\left|\phi_{n}(x, y)\right|$ for all $(x, y) \in B_{1}, 0 \leq t<T$.

Proof. Let $\Omega^{\prime}$ denote the wedge $\left\{(r, \theta): 0<r<1, \theta \in\left(-\frac{\pi}{2 n}, \frac{\pi}{2 n}\right)\right\}$ in $(r, \theta)$ polar coordinates. The solution $u(x, y, t)$ will possess, for all $0<t<T$, the same symmetries (24)-(25) as $\phi_{n}$. Given the symmetry of $u$ it suffices to prove the estimate $|u(x, y, t)| \leq$ $m\left|\phi_{n}(x, y)\right|$ on $\Omega^{\prime}$ and for all $0<t<T$. Note that $\phi_{n}(x, y)>0$ in $\Omega^{\prime}$. Let $\partial \Omega_{1}^{\prime}$ denote the portion of $\partial \Omega^{\prime}$ that consists of the two straight edges $\left\{(r, \theta): 0 \leq r \leq 1, \theta= \pm \frac{\pi}{2 n}\right\}$ (including their endpoints) and let $\partial \Omega_{2}^{\prime}$ denote the remaining part of $\partial \Omega^{\prime}$, namely the open circular $\operatorname{arc}\left\{(r, \theta): r=1, \theta \in\left(-\frac{\pi}{2 n}, \frac{\pi}{2 n}\right)\right\}$. Both $f \equiv 0$ and $u(\cdot, t) \equiv 0$ on $\partial \Omega_{1}^{\prime}$. Choose $m^{\prime} \in(m, 1)$ and let $\phi(x, y)=m^{\prime} \phi_{n}(x, y)$. Then

$$
\frac{\partial \phi}{\partial \mathbf{n}}(x, y)=m^{\prime} \frac{\partial \phi_{n}}{\partial \mathbf{n}}(x, y)=m^{\prime} \lambda F\left(\phi_{n}(x, y)\right)>\lambda F(\phi(x, y))
$$

on $\partial \Omega_{2}^{\prime}$. Here we use that $\phi_{n}(x, y)>0$ on $\partial \Omega_{2}^{\prime}$, and that $F(z) / z, z>0$, is strictly increasing, so $F\left(m^{\prime} z\right) /\left(m^{\prime} z\right)<F(z) / z$ for $m^{\prime}<1$ and $z>0$, implying $F\left(m^{\prime} z\right)<m^{\prime} F(z)$.

Define $v=\phi-u$, so $v$ satisfies the heat equation and has the same regularity as $u$, and symmetries (24)-(25). We have $v(x, y, 0)>0$ in $\Omega^{\prime}$ and $v \equiv 0$ on $\partial \Omega_{1}^{\prime}$. Also, from (29) we obtain

$$
\begin{aligned}
\frac{\partial v}{\partial \mathbf{n}}(x, y, t) & =\frac{\partial \phi}{\partial \mathbf{n}}(x, y)-\frac{\partial u}{\partial \mathbf{n}}(x, y, t) \\
& >\lambda F(\phi(x, y))-\lambda F(u(x, y, t))
\end{aligned}
$$

for $(x, y) \in \partial \Omega_{2}^{\prime}$ and $0 \leq t<T$. For each $(x, y)$ in $\partial \Omega_{2}^{\prime}$ we define $\tau(x, y)=\sup \{0<t<$ $T: v(x, y, s) \geq 0$ for all $s \in[0, t]\}$. Since $u(x, y, 0)=f(x, y)<m^{\prime} \phi_{n}(x, y)=\phi(x, y)$ for $(x, y) \in \partial \Omega_{2}^{\prime}$ we conclude by continuity that $\tau(x, y)>0$ for any $(x, y) \in \partial \Omega_{2}^{\prime}$. Let

$$
t^{*}=\inf \left\{\tau(x, y):(x, y) \in \partial \Omega_{2}^{\prime}\right\}
$$

Claim 1. $t^{*}>0$.

To prove this, first note that $\tau(x, y)>0$ for any $(x, y) \in \partial \Omega_{2}^{\prime}$, and $\tau(x, y)$ is uniformly bounded away from 0 on any compact subset of $\partial \Omega_{2}^{\prime}$. Let $p$ denote the corner point of $\Omega^{\prime}$ with polar coordinates $r=1, \theta=\alpha$, where $\alpha=-\frac{\pi}{2 n}$, and consider $(1, \theta)$ in a neighborhood $S=\partial \Omega_{2}^{\prime} \cap B_{\epsilon}(p)$ of $p$, for some $\epsilon>0$. We have

$$
\begin{aligned}
v(1, \theta, t) & =v(1, \alpha, t)+v_{\theta}(1, \alpha, t)(\theta-\alpha)+\frac{1}{2} v_{\theta \theta}(1, z, t)(\theta-\alpha)^{2} \\
& =v_{\theta}(1, \alpha, t)(\theta-\alpha)+\frac{1}{2} v_{\theta \theta}(1, z, t)(\theta-\alpha)^{2}
\end{aligned}
$$


since $v(1, \alpha, t)=0$ for all $t \geq 0$. The value $z$ lies between $\alpha=-\frac{\pi}{2 n}$ and $\theta$. From $v=m^{\prime} \phi_{n}-u$ we obtain

$$
v_{\theta}(1, \alpha, 0)=m^{\prime}\left(\phi_{n}\right)_{\theta}(1, \alpha)-f_{\theta}(1, \alpha) .
$$

It's easy to check that $\left(\phi_{n}\right)_{\theta}(1, \alpha)>0$, and since $\phi_{n}(1, \alpha)=f(1, \alpha)=0$ and $|f| \leq m \phi_{n}$ on $\partial \Omega_{2}^{\prime}$ we have

$$
\left|f_{\theta}(1, \alpha)\right| \leq m\left(\phi_{n}\right)_{\theta}(1, \alpha)<m^{\prime}\left(\phi_{n}\right)_{\theta}(1, \alpha)
$$

for any $m^{\prime}>m$. We conclude from (32) and (33) that $v_{\theta}(1, \alpha, 0)>0$. From the continuity of $v_{\theta}$ it follows that there exists $\delta_{1}>0$ and $\delta_{2}>0$ so that

$$
d=\min _{(\theta, t) \in\left[\alpha, \alpha+\delta_{1}\right] \times\left[0, \delta_{2}\right]} v_{\theta}(1, \theta, t)>0 .
$$

Let $M$ denote an upper bound for $\left|v_{\theta \theta}(1, z, t)\right|$. For $\theta \in\left[\alpha, \alpha+\delta_{1}\right], t \in\left[0, \delta_{2}\right]$ we can now use (31) to estimate

$$
\begin{aligned}
v(1, \theta, t) & \geq v_{\theta}(1, \alpha, t)(\theta-\alpha)-M(\theta-\alpha)^{2} / 2 \\
& =(\theta-\alpha)\left(v_{\theta}(1, \alpha, t)-M(\theta-\alpha) / 2\right) \\
& \geq(\theta-\alpha)\left(v_{\theta}(1, \alpha, t)-M \delta_{1} / 2\right)
\end{aligned}
$$

with $M$ independent of $\delta_{1}, \delta_{2}$ and of $\theta$ and $t$ in the specified neighborhoods of $\alpha$ and 0 . By selecting $\delta_{1}$ so that $M \delta_{1} / 2<d$, where $d$ is the minimum introduced above, we obtain that

$$
v(1, \theta, t) \geq 0 \text { for } \theta \in\left[\alpha, \alpha+\delta_{1}\right], t \in\left[0, \delta_{2}\right],
$$

which implies that $\tau(x, y)$ is greater than or equal to $\delta_{2}$ in a $\partial \Omega_{2}^{\prime}$ neighborhood of the corner point $r=1, \theta=-\frac{\pi}{2 n}$. The same fact holds in a $\partial \Omega_{2}^{\prime}$ neighborhood of the corner point $r=1, \theta=\frac{\pi}{2 n}$. We may thus conclude that $\tau(x, y)$ is bounded uniformly away from 0 on $\partial \Omega_{2}^{\prime}$, which establishes Claim 1 .

Claim 2. $t^{*}=T$.

To prove this, let us assume to the contrary that $t^{*}<T$. Let $q_{l}$ denote a "minimizing" sequence for $\tau$; in other words,

$$
q_{l} \in \partial \Omega_{2}^{\prime} \text { and } \tau\left(q_{l}\right) \rightarrow t^{*} \text { as } l \rightarrow \infty .
$$

After extraction of a subsequence there are now two possibilities:

$$
\text { (i) : } q_{l} \rightarrow q \in \partial \Omega_{2}^{\prime},
$$

or $q_{l}$ converges to one of the endpoints of $\partial \Omega_{2}^{\prime}$, say;

$$
\text { (ii) : } q_{l} \rightarrow p \text {, where } p=(1, \alpha) \text { in polar coordinates, with } \alpha=-\frac{\pi}{2 n} \text {. }
$$

Let us start by considering the possibility $(i)$. In this case it is easy to see that

$$
0=v\left(q_{l}, \tau\left(q_{l}\right)\right) \rightarrow v\left(q, t^{*}\right),
$$

and so $v\left(q, t^{*}\right)=0$, with $q \in \partial \Omega_{2}^{\prime}$. Due to the maximum principle (Theorem 5, Section 3 of Chapter 3 in [23]), $\inf _{\Omega^{\prime} \times\left[0, t^{*}\right]} v=0$ and so the fact that $v\left(q, t^{*}\right)=0$ implies that $\frac{\partial v}{\partial \mathbf{n}}\left(q, t^{*}\right) \leq 0$. However, due to (30) and the fact that $u\left(q, t^{*}\right)=\phi(q)\left(\right.$ since $\left.v\left(q, t^{*}\right)=0\right)$ we also have

$$
\frac{\partial v}{\partial \mathbf{n}}\left(q, t^{*}\right)>0,
$$


a clear contradiction. We may conclude that $(i)$ is not possible, and so we are left with the possibility (ii), i.e.,

$$
q_{l} \rightarrow p \text {, where } p=(1, \alpha) \text { in polar coordinates, with } \alpha=-\frac{\pi}{2 n} .
$$

We have that

$$
v\left(1, \alpha, t^{*}\right)=0, \text { and that } v\left(1, \theta, t^{*}\right) \geq 0 \text { for } \theta \in(\alpha,-\alpha),
$$

with $\alpha=-\frac{\pi}{2 n}$. Since $v$ is $C^{N+1}$ in $\theta$ we may write

$$
v\left(1, \theta, t^{*}\right)=\sum_{k=0}^{N} \frac{\partial^{k} v}{\partial \theta^{k}}\left(1, \alpha, t^{*}\right) \frac{(\theta-\alpha)^{k}}{k !}+\frac{\partial^{N+1} v}{\partial \theta^{N+1}}\left(1, z, t^{*}\right) \frac{(\theta-\alpha)^{N+1}}{(N+1) !},
$$

for $\theta>\alpha$ and $z \in(\alpha, \theta)$. Due to the odd symmetry of $u, \phi_{n}$, and hence $v$ about $\theta=\alpha$ we have

$$
\frac{\partial^{k} v}{\partial \theta^{k}}(1, \alpha, t)=0
$$

for all nonnegative even integers $k$ and all $t \geq 0$. Thus the sum on the right in (36) contains only terms of odd index. Consider now (36) in the case $N=1$, which yields

$$
v\left(1, \theta, t^{*}\right)=v_{\theta}\left(1, \alpha, t^{*}\right)(\theta-\alpha)+\frac{1}{2} v_{\theta \theta}\left(1, z, t^{*}\right)(\theta-\alpha)^{2} .
$$

Due to (35) it follows that $v_{\theta}\left(1, \alpha, t^{*}\right) \geq 0$. However, if $v_{\theta}\left(1, \alpha, t^{*}\right)>0$, then an argument almost identical to that following equation (34) shows that $\tau(q) \geq t^{*}+\epsilon$ for some $\epsilon>0$ and for all $q$ in a $\partial \Omega_{2}^{\prime}$ neighborhood of $p$. This contradicts the fact that $q_{l}$ is a minimizing sequence with $q_{l} \rightarrow p$. We must conclude that $v_{\theta}\left(1, \alpha, t^{*}\right)=0$, and therefore that the first nonzero term in the sum on the right in (36) is of index $k \geq 3$. The exact same argument given above now yields $\frac{\partial^{3} v}{\partial \theta^{3}}\left(1, \alpha, t^{*}\right)=0$, and indeed we find that all derivatives $\frac{\partial^{k} v}{\partial \theta^{k}}\left(1, \alpha, t^{*}\right)$ with $k$ odd must also equal zero. Since $v\left(1, \theta, t^{*}\right)$ is analytic in $\theta$ it follows that $v\left(1, \theta, t^{*}\right)=0$ for $\theta$ in a neighborhood of $\theta=\alpha$; in other words, there are points $q \in \partial \Omega_{2}^{\prime}$ with $v\left(q, t^{*}\right)=0$. However, the existence of such points was (due to the maximum principle) ruled out above. We conclude that (ii) is not possible either, and thus it follows that $t^{*}<T$ cannot be true. This completes the proof of Claim 2.

To complete the proof of the lemma, note that $t^{*}=T$ implies that $v(x, y, t) \geq 0$ for $(x, y) \in \overline{\partial \Omega_{2}^{\prime}}$ and all $0 \leq t<T$. The maximum principle (and the fact that $v(x, y, 0)>0$ for $\left.(x, y) \in \Omega^{\prime}\right)$ then implies that $v(x, y, t)>0$ for $(x, y) \in \Omega^{\prime}$ and all $0 \leq t<T$, so that $u \leq m^{\prime} \phi_{n}$ on $\overline{\Omega^{\prime}} \times[0, T)$, for any $m^{\prime}>m$. This implies that $u \leq m \phi_{n}$ on $\overline{\Omega^{\prime}} \times[0, T)$. A similar argument shows that $-m \phi_{n} \leq u$ on $\overline{\Omega^{\prime}} \times[0, T)$, and this completes the proof of Lemma 3.1 .

Using the same techniques as in the proof of Lemma 3.1 we may also prove the following result.

Lemma 3.2. Let $\phi_{n}$ denote the steady-state solution defined by (23). Let $u(x, y, t), 0 \leq$ $t<T$, be a classical solution to the diffusion problem (26)-(28), with initial condition $f$ satisfying the same symmetry conditions (24)-(25) as $\phi_{n}$, and the compatibility condition $\frac{\partial f}{\partial \mathbf{n}}=\lambda F(f)=\lambda \sinh (f)$ on $\partial B_{1}$. If the initial condition additionally satisfies $|f(x, y)| \geq$ 
$m\left|\phi_{n}(x, y)\right|,(x, y) \in B_{1}$, for some $m>1$, then $|u(x, y, t)| \geq m\left|\phi_{n}(x, y)\right|$ for all $(x, y) \in$ $B_{1}, 0 \leq t<T$.

3.2. Decay. We now proceed to establish a decay result similar to that from one space dimension. In order to do so we need some information about solutions to a related linear problem. Given any $m>0$ and any $\theta \in\left(-\frac{\pi}{2 n}, \frac{\pi}{2 n}\right)$ we define

$$
B(m, \theta)=\frac{\lambda F\left(m \phi_{n}(1, \theta)\right)}{m \phi_{n}(1, \theta)}=\frac{\lambda \sinh \left(m \phi_{n}(1, \theta)\right)}{m \phi_{n}(1, \theta)} .
$$

Note that for any fixed $\theta$ the function $B(m, \theta)>0$ is strictly increasing in $m>0$. Let $v$ denote the solution to the linear initial-boundary value problem

$$
\begin{aligned}
v_{t}-\Delta v & =0 \text { in } \Omega^{\prime} \times(0, \infty), \\
\frac{\partial v}{\partial \mathbf{n}} & =B(m, \theta) v \text { on } \partial \Omega_{2}^{\prime} \times(0, \infty), \\
v & =0 \text { on } \partial \Omega_{1}^{\prime} \times(0, \infty) \\
v(x, y, 0) & =m \phi_{n}(x, y) \text { in } \Omega^{\prime} .
\end{aligned}
$$

A standard separation of variables shows that $v$ can be written as

$$
v(x, y, t)=\sum_{k=0}^{\infty} A_{k} e^{-\mu_{k} t} \psi_{k}(x, y),
$$

where the $\psi_{k}$ and $\mu_{k}$ satisfy

$$
\begin{aligned}
\Delta \psi_{k} & =-\mu_{k} \psi_{k} \text { in } \Omega^{\prime}, \\
\frac{\partial \psi_{k}}{\partial \mathbf{n}} & =B(m, \theta) \psi_{k} \text { on } \partial \Omega_{2}^{\prime}, \\
\psi_{k} & =0 \text { on } \partial \Omega_{1}^{\prime} .
\end{aligned}
$$

That is, the $\psi_{k}, \mu_{k}$ are the eigenfunctions and eigenvalues for the Laplacian with the stated boundary conditions, and the $\psi_{k}$ form an orthogonal basis for $L^{2}\left(\Omega^{\prime}\right)$. We note that $\psi_{k}$ and $\mu_{k}$ depend on $m, n$, and $\lambda$, though we do not explicitly indicate this. The functions $\psi_{k}$ may be thought of as eigenfunctions on all of $B_{1}$ with special symmetries. For that reason it is not very difficult to see that they are all infinitely smooth, in spite of the fact that $\Omega^{\prime}$ has "corners". The $A_{k}$ are determined as the $L^{2}\left(\Omega^{\prime}\right)$ inner products $A_{k}=\left\langle f, \psi_{k}\right\rangle$ if we normalize the $\psi_{k}$ to have $L^{2}\left(\Omega^{\prime}\right)$ norm 1. As shown in [8] (Courant and Hilbert, Chapter 6 , section 5) the $\mu_{k}$ are all real and limit to infinity as $k \rightarrow \infty$, and only a finite number of the $\mu_{k}$ are negative. We assume they are ordered as

$$
\mu_{0} \leq \mu_{1} \leq \mu_{2} \leq \cdots .
$$

It is possible to characterize the $\mu_{k}$ variationally, in particular $\mu_{0}=\inf _{\psi} Q(m, \psi)$, where

$$
Q(m, \psi)=\frac{\int_{\Omega^{\prime}}|\nabla \psi|^{2} d x-\int_{\partial \Omega_{2}^{\prime}} B(m, \theta) \psi^{2} d s}{\int_{\Omega^{\prime}} \psi^{2} d x},
$$

and where $\psi$ ranges over the class of $C^{1}\left(\overline{\Omega^{\prime}}\right)$ functions that satisfy $\psi \equiv 0$ on $\partial \Omega_{1}^{\prime}$.

We shall need the following fact about $\mu_{0}$. 
LEMmA 3.3. Let $\mu_{0}$ denote the principal eigenvalue introduced above. Then

$$
\mu_{0}>0 \text { if } 0<m<1, \mu_{0}=0 \text { if } m=1 \text {, and } \mu_{0}<0 \text { if } m>1 \text {. }
$$

Proof. Consider first the case $m=1$. In this case the function $\phi_{n}$ is an eigenfunction for (41) with eigenvalue 0 . If 0 was not the principal eigenvalue, then $\phi_{n}$ would have to be $L^{2}\left(\Omega^{\prime}\right)$-orthogonal to any principal eigenfunction. A standard argument (see Courant and Hilbert, Chapter 6, section 6) shows that any principal eigenfunction must be of one sign, and since $\phi_{n}$ is also of one sign, orthogonality is impossible. We therefore conclude that $\mu_{0}=0$ for $m=1$. For $m \neq 1$ the monotonicity of $B(m, \theta)$ implies that

$$
Q(m, \psi)>Q(1, \psi) \text { if } 0<m<1 \text { and } \psi \text { does not identically vanish on } \partial \Omega_{2}^{\prime},
$$

and

$$
Q(m, \psi)<Q(1, \psi) \text { if } m>1 \text { and } \psi \text { does not identically vanish on } \partial \Omega_{2}^{\prime} \text {. }
$$

Since no eigenfunction of (41) can vanish identically on any open portion of $\partial \Omega_{2}^{\prime}$ (by unique continuation it would then have to vanish identically in $\Omega^{\prime}$ ) the above two inequalities and the fact that $\mu_{0}=0$ for $m=1$ now give that $\mu_{0}>0$ for $0<m<1$, and $\mu_{0}<0$ for $m>1$, respectively.

We are now ready to prove the decay result.

Proposition 3.4. Let $u(x, y, t), 0 \leq t<\infty$, be a globally defined, classical solution to the diffusion problem (26)-(28), with initial condition $f$. Let the assumptions concerning $f$ be as in Lemma 3.1 in particular, assume $|u(x, y, 0)|=|f(x, y)| \leq m\left|\phi_{n}(x, y)\right|,(x, y) \in$ $B_{1}$, for some $0<m<1$. Then

$$
\lim _{t \rightarrow \infty} u(x, y, t)=0
$$

uniformly with respect to $(x, y) \in B_{1}$.

Proof. We again focus on the wedge $\Omega^{\prime}$ and use $(r, \theta)$ to indicate polar coordinates when convenient. From Lemma 3.1 we have $|u(x, y, t)| \leq m \phi_{n}(x, y)$ for all $t$. We may subject this function to a maximum principle argument very similar to that used on the function $v$ in the proof of Lemma 3.1 .

Choose $m^{\prime}$ such that $m<m^{\prime}<1$ and let $v^{\prime}$ denote the solution to the linear problem (39) with $m$ replaced by $m^{\prime}$. The function $w=v^{\prime}-u$ satisfies the heat equation in $\Omega^{\prime} \times(0, \infty)$ with $w(x, y, 0)=m^{\prime} \phi_{n}(x, y)-f(x, y) \geq\left(m^{\prime}-m\right) \phi_{n}(x, y)>0$ in $\Omega^{\prime}$. Also, $w=0$ on $\partial \Omega_{1}^{\prime} \times(0, \infty)$, and

$$
\begin{aligned}
\frac{\partial w}{\partial \mathbf{n}} & =\frac{\partial v^{\prime}}{\partial \mathbf{n}}-\frac{\partial u}{\partial \mathbf{n}} \\
& =\lambda B\left(m^{\prime}, \theta\right) v^{\prime}-\lambda F(u)
\end{aligned}
$$

on $\partial \Omega_{2}^{\prime} \times(0, \infty)$. At a point on $\partial \Omega_{2}^{\prime} \times(0, \infty)$ where $w=0$ (and thus $u=v^{\prime}>0$ ) it follows that $0<u \leq m \phi_{n}<m^{\prime} \phi_{n}$, and thus

$$
\lambda F(u)<\frac{\lambda F\left(m^{\prime} \phi_{n}\right)}{m^{\prime} \phi_{n}} u=B\left(m^{\prime}, \theta\right) u .
$$


As a consequence of this and the previous identity,

$$
\frac{\partial w}{\partial \mathbf{n}}>\lambda B\left(m^{\prime}, \theta\right) v^{\prime}-\lambda B\left(m^{\prime}, \theta\right) u=0
$$

at any point on $\partial \Omega_{2}^{\prime} \times(0, \infty)$ where $w=0$. A maximum principle argument (somewhat complicated by the presence of the "corners" of $\Omega^{\prime}$ on $\partial \Omega_{2}^{\prime}$, but) similar to that of Lemma 3.1 now shows that

$$
w>0 \text { on } \partial \Omega_{2}^{\prime}, \text { and } w>0 \text { in } \Omega^{\prime} \text { for all } t \geq 0,
$$

and therefore

$$
u \leq v^{\prime} \text { on } \overline{\Omega^{\prime}} \text { for all } t \geq 0 .
$$

An entirely similar argument establishes that $-v^{\prime} \leq u$ on $\overline{\Omega^{\prime}}$, for all $t \geq 0$. From the formula (40) and Lemma 3.3 we have that $\lim _{t \rightarrow \infty} v^{\prime}(x, y, t)=0$ uniformly with respect to $(x, y) \in B_{1}$, and in conjunction with the above estimates we see that $\lim _{t \rightarrow \infty} u(x, y, t)=$ 0 , uniformly with respect to $(x, y) \in \overline{\Omega^{\prime}}$ (and in $B_{1}$ ). This concludes the proof of Proposition 3.4 .

3.3. Growth and blowup in two dimensions. We now establish an alternative to Proposition 3.4 for the case when $|u(x, y, 0)| \geq m\left|\phi_{n}(x, y)\right|$, for some $m>1$. In order to do so we shall need a result comparing the special solution $\phi_{n}$ defined in equation (23) to the principal eigenfunction $\psi_{0}$ of (41). Even though it is not explicitly expressed in the notation, $\psi_{0}$ depends on $m, n$, and $\lambda$.

Lemma 3.5. There exist positive constants $c_{1}, c_{2}$, depending on $m>1, n$ and $\lambda$, so that

$$
c_{1} \leq \frac{\psi_{0}(x, y)}{\phi_{n}(x, y)} \leq c_{2}
$$

for all $(x, y) \in \Omega^{\prime}$.

Proof. Note that both functions $\psi_{0}$ and $\phi_{n}$ are positive away from $\partial \Omega_{1}^{\prime}$, so if the ratio approaches zero or is unbounded, then this behavior occurs at $\partial \Omega_{1}^{\prime}$. Let us first show that $\psi_{0} / \phi_{n}$ is bounded. The smooth function $\psi_{0}$ vanishes on $\partial \Omega_{1}^{\prime}$, and so does $\phi_{n}$; moreover, it's easy to check that $\frac{\partial \phi_{n}}{\partial \mathbf{n}}=\frac{\partial \phi_{n}}{\partial \theta} \neq 0$ on $\partial \Omega_{1}^{\prime}$ away from the origin, so clearly the ratio $\psi_{0} / \phi_{n}$ will remain bounded up to $\partial \Omega_{1}^{\prime}$ away from the origin. At the origin it's easy to check that $\phi_{n}$ vanishes to exactly order $n$ (for $r^{n}$ near $r=0$ ). Given the symmetry of $\psi_{0}$ (as a function on the entire disk) the function $\psi_{0}$ must vanish to at least order $n$. To see this we simply expand $\psi_{0}(r, \theta)$ in terms of the orthogonal basis functions $\eta_{j}(r, \theta)=\cos (n j \theta) I_{n j}\left(\sqrt{-\mu_{0}} r\right), j \geq 1$, where $I_{k}$ denotes the modified Bessel function of order $k$, and we note that $I_{k}$ vanishes to order $k$ at $r=0$. Thus the ratio $\psi_{0} / \phi_{n}$ is bounded near the origin too, and we infer the existence of a constant $c_{2}$ so that $\psi_{0}(x, y) / \phi_{n}(x, y) \leq c_{2}$ for $(x, y) \in \Omega^{\prime}$.

A similar argument works to show that there is a positive constant $c_{1}$ such that $c_{1} \leq \psi_{0} / \phi_{n}$ on $\Omega^{\prime}$. First note that $\psi_{0}$ in fact vanishes to exactly order $n$ at the origin, for if not, then $\psi_{0}$ could be expanded in terms of the functions $\eta_{j}(r, \theta)=\cos (n j \theta) I_{n j}\left(\sqrt{-\mu_{0}} r\right)$ for $j \geq 2$ (the term corresponding to $\eta_{1}(r, \theta)$ would be absent) and so $\psi_{0}$ would vanish at points inside $\Omega^{\prime}$, a contradiction, since $\psi_{0}$ is strictly positive in $\Omega^{\prime}$. Since $\phi_{n}$ vanishes to precisely order $n$ we conclude that $\psi_{0} / \phi_{n}$ is bounded away from 0 near the origin. 
Secondly, we claim that $\frac{\partial \psi_{0}}{\partial \mathbf{n}} \neq 0$ at any point $p \in \partial \Omega_{1}^{\prime}$, away from the origin. For if $\frac{\partial \psi_{0}}{\partial \mathbf{n}}(p)=0$, then an argument similar to that of Lemma 1.2 in [3] shows that there must be a curve $C$ such that $C \cap \partial \Omega_{1}^{\prime}=p$ and $\psi_{0} \equiv 0$ on $C$ (in this case the argument can be based on an expansion into functions of the form $e^{i k \theta} I_{k}\left(\sqrt{-\mu_{0}} r\right)$ rather than $e^{i k \theta} r^{k}$, where $(r, \theta)$ denote polar coordinates around the point $p)$. However, the existence of such a curve $C$ contradicts the fact that $\psi_{0}$ cannot vanish in $\Omega^{\prime}$. We conclude that $\psi_{0} / \phi_{n}$ does not vanish anywhere on (or near) $\partial \Omega_{1}^{\prime}$, and thus the first inequality of (43) also holds. This verifies Lemma 3.5 .

We are now ready to show that any classical (real analytic) solution to the nonlinear diffusion problem with initial datum $|u(x, y, 0)| \geq m\left|\phi_{n}(x, y)\right|, m>1$, will either cease to exist in finite time, or grow exponentially (at an arbitrary rate) as time approaches infinity.

Proposition 3.6. Let $\phi_{n}$ denote the steady-state solution defined by (23). Let $u(x, y, t)$, $0 \leq t<\infty$, be a globally defined, classical solution to the diffusion problem (26)-(27), with initial condition $f$ that possesses the same symmetry properties (24)-(25) as $\phi_{n}$, and satisfies the compatibility condition $\frac{\partial f}{\partial \mathbf{n}}=\lambda F(f)=\lambda \sinh (f)$ on $\partial B_{1}$. If additionally $|f(x, y)| \geq m\left|\phi_{n}(x, y)\right|,(x, y) \in B_{1}$, for some $m>1$, then

$\forall K \in \mathbb{R} \exists c_{K}>0$ such that $|u(x, y, t)| \geq c_{K} e^{K t}\left|\phi_{n}(x, y)\right| \forall(x, y) \in B_{1}, 0 \leq t<\infty$.

Proof. As in the proof of Lemma 3.1, we may confine our attention to the wedge $\Omega^{\prime}$. Let $m^{\prime} \in(1, m)$ be fixed, and define $B\left(m^{\prime}, \theta\right)$ as in equation (38). From Lemma 3.2 we know that $|u(x, y, t)| \geq m \phi_{n}(x, y)>m^{\prime} \phi_{n}(x, y)$ for all $t$ and all $(x, y) \in \Omega^{\prime}$; suppose for now that $u(x, y, t) \geq m \phi_{n}(x, y)$. As a result, $\lambda \frac{F(u)}{u}>\lambda \frac{F\left(m^{\prime} \phi_{n}\right)}{m^{\prime} \phi_{n}}=B\left(m^{\prime}, \theta\right)$ on $\partial \Omega_{2}^{\prime}$, so that

$$
\frac{\partial u}{\partial \mathbf{n}}=B\left(m^{\prime}, \theta\right) u+h(\theta)
$$

where $h(\theta)>0,-\frac{\pi}{2 n}<\theta<\frac{\pi}{2 n}$.

Let $\psi_{0}^{\prime}, \mu_{0}^{\prime}$ denote the eigenvector-eigenvalue pair from (41), corresponding to $\mathrm{m}^{\prime}$, and let $c_{1}^{\prime}, c_{2}^{\prime}$ be the appropriate constants from Lemma 3.5. Define

$$
v^{\prime}(x, y, t)=\frac{m^{\prime}}{c_{2}^{\prime}} e^{-\mu_{0}^{\prime} t} \psi_{0}^{\prime}(x, y) .
$$

Note that $\mu_{0}^{\prime}<0$ according to Lemma 3.3, and so $v^{\prime}$ grows exponentially in $t$. Due to Lemma 3.5,

$$
\frac{m^{\prime}}{c_{2}^{\prime}} \psi_{0}^{\prime}(x, y) \leq m^{\prime} \phi_{n}(x, y),
$$

for all $(x, y) \in \Omega^{\prime}$, which immediately yields

$$
v^{\prime}(x, y, 0) \leq m^{\prime} \phi_{n}(x, y)<m \phi_{n}(x, y) \leq u(x, y, 0) \text { for all }(x, y) \in \Omega^{\prime} .
$$

The function $v^{\prime}$ also satisfies $v^{\prime} \equiv 0$ on $\partial \Omega_{1}^{\prime}$, and $\frac{\partial v^{\prime}}{\partial \mathbf{n}}=B\left(m^{\prime}, \theta\right) v^{\prime}$ on $\partial \Omega_{2}^{\prime}$. In view of equation (44) a comparison argument (as in the proof of Lemma 3.1) shows that $v^{\prime}(x, y, t) \leq u(x, y, t)$ for all $x, y, t$. From (45) and Lemma 3.5 we obtain

$$
\frac{m^{\prime} c_{1}^{\prime}}{c_{2}^{\prime}} e^{-\mu_{0}^{\prime} t} \phi_{n}(x, y) \leq v^{\prime}(x, y, t) \leq u(x, y, t) \text {. }
$$


This makes it clear that for any $M>1$ we may find a $T>0$ so that

$$
M \phi_{n}(x, y)<u(x, y, t)
$$

for all $(x, y) \in \Omega^{\prime}$ and $t \geq T$. We conclude that for any $(x, y) \in \Omega^{\prime}$ we have that $\lim _{t \rightarrow \infty} u(x, y, t)=\infty$ (under the assumption that the solution exists for all $t$ ).

We can estimate the growth rate of $v^{\prime}$ (and hence $u$ ) by estimating the eigenvalue $\mu_{0}(\tilde{m})$ corresponding to $\tilde{m}$. With $Q$ as defined by equation (42) we have

$$
\mu_{0}(\tilde{m})=\inf _{\psi} Q(\tilde{m}, \psi) \leq Q\left(\tilde{m}, \phi_{n}\right),
$$

where $\psi$ ranges over the class of $C^{1}\left(\overline{\Omega^{\prime}}\right)$ functions that satisfy $\psi \equiv 0$ on $\partial \Omega_{1}^{\prime}$ (note that $\phi_{n}$ satisfies this boundary condition). According to (22), for any $\gamma<1$,

$$
B(\tilde{m}, \theta)=\frac{\lambda F\left(\tilde{m} \phi_{n}\right)}{\tilde{m} \phi_{n}} \geq c e^{\gamma \tilde{m} \phi_{n}} \text { on } \partial \Omega_{2}^{\prime},
$$

for some $c>0$. We thus have

$$
\begin{aligned}
Q\left(\tilde{m}, \phi_{n}\right) & =\frac{\int_{\Omega^{\prime}}\left|\nabla \phi_{n}\right|^{2} d x-\int_{\partial \Omega_{2}^{\prime}} \frac{\lambda F\left(\tilde{m} \phi_{n}\right)}{\tilde{m} \phi_{n}} \phi_{n}^{2} d s}{\int_{\Omega^{\prime}} \phi_{n}^{2} d x} \\
& \leq \frac{\int_{\Omega^{\prime}}\left|\nabla \phi_{n}\right|^{2} d x-c e^{\gamma \tilde{m} \delta} \int_{\partial \Omega_{2}^{\prime} \cap\left\{\phi_{n}>\delta\right\}} \phi_{n}^{2} d s}{\int_{\Omega^{\prime}} \phi_{n}^{2} d x} \\
& =C_{1}-c_{2} e^{\gamma \tilde{m} \delta}
\end{aligned}
$$

for any $\gamma<1$, and any $\delta$ strictly inside the range of $\phi_{n}$. The constants $C_{1}, c_{2}$ are positive, and independent of $\tilde{m}$. It follows that $\mu_{0}(\tilde{m}) \leq C_{1}-c_{2} e^{\gamma \tilde{m} \delta}$, or

$$
-\mu_{0}(\tilde{m}) \geq c_{2} e^{\gamma \tilde{m} \delta}-C_{1} .
$$

Note that $-\mu_{0}(\tilde{m})>0$ for any $\tilde{m}>1$, though the above estimate doesn't yield that inequality unless $\tilde{m}$ is sufficiently large. The estimate (48) shows that

$$
-\mu_{0}(\tilde{m}) \rightarrow \infty \text { as } \tilde{m} \rightarrow \infty \text {. }
$$

Now suppose that $M>2$ is chosen so that $-\mu_{0}(\tilde{m}) \geq K$ for $\tilde{m}>M / 2$, and that $T$ is chosen so that (47) holds. Instead of $u$ we now consider $\tilde{u}(x, y, t)=u(x, y, T+t) ; \tilde{u}$ solves the same diffusion problem as $u$, only with an initial condition that is bounded below by $M \phi_{n}(x, y)$. From (46) it follows that for any $1<\tilde{m}<M$,

$$
\frac{\tilde{m} \tilde{c}_{1}}{\tilde{c}_{2}} e^{-\mu_{0}(\tilde{m}) t} \phi_{n}(x, y) \leq \tilde{u}(x, y, t)=u(x, y, T+t), \forall(x, y) \in \Omega^{\prime}, \forall t>0 .
$$

By choosing a fixed $\tilde{m}$ with $M / 2<\tilde{m}<M$ we thus obtain

$$
c_{K} e^{K t} \phi_{n}(x, y) \leq u(x, y, t), \forall(x, y) \in \Omega^{\prime}, \forall t>T .
$$

From (46) we know that the same inequality holds for $0<t \leq T$. This concludes the proof of Proposition 3.6 in case $u(x, y, t) \geq m \phi_{n}(x, y)$. The alternative case $u(x, y, t) \leq$ $-m \phi_{n}(x, y)$ can be treated with a similar argument. 
We proceed to use the energy criterion (explained in detail in Appendix 4) in combination with Proposition 3.6 to show that solutions to the diffusion problem (26)-(27), with initial data that pointwise "exceed" one of the steady state solutions $\phi_{n}$, can only exist for a finite period of time. For this purpose the following lemma will prove very useful.

Lemma 3.7. There exists a constant $C$ such that

$$
\|v\|_{L^{2}\left(B_{1}\right)}^{2} \leq C\left(\|\Delta v\|_{L^{2}\left(B_{1}\right)}^{2}+\|v\|_{L^{2}\left(\partial B_{1}\right)}^{2}\right),
$$

for any $v \in H^{2}\left(B_{1}\right)$.

Proof. Let $\psi$ be the solution to

$$
\Delta \psi=v \text { in } B_{1}, \text { with } \psi=0 \text { on } \partial B_{1} .
$$

Standard elliptic estimates assert that

$$
\|\psi\|_{H^{2}\left(B_{1}\right)} \leq C\|v\|_{L^{2}\left(B_{1}\right)} .
$$

Furthermore, due to Green's formula,

$$
\begin{aligned}
\int_{B_{1}} v^{2} d x d y & =\int_{B_{1}} v \Delta \psi d x d y \\
& =\int_{B_{1}} \psi \Delta v d x d y+\int_{\partial B_{1}} v \frac{\partial \psi}{\partial \mathbf{n}} d \sigma
\end{aligned}
$$

and so

$$
\begin{aligned}
\|v\|_{L^{2}\left(B_{1}\right)}^{2} & \leq\|\Delta v\|_{L^{2}\left(B_{1}\right)}\|\psi\|_{L^{2}\left(B_{1}\right)}+\|v\|_{L^{2}\left(\partial B_{1}\right)}\|\partial \psi / \partial \mathbf{n}\|_{L^{2}\left(\partial B_{1}\right)} \\
& \leq C\left(\|\Delta v\|_{L^{2}\left(B_{1}\right)}+\|v\|_{L^{2}\left(\partial B_{1}\right)}\right)\|\psi\|_{H^{2}\left(B_{1}\right)} \\
& \leq C\left(\|\Delta v\|_{L^{2}\left(B_{1}\right)}+\|v\|_{L^{2}\left(\partial B_{1}\right)}\right)\|v\|_{L^{2}\left(B_{1}\right)}
\end{aligned}
$$

or

$$
\|v\|_{L^{2}\left(B_{1}\right)} \leq C\left(\|\Delta v\|_{L^{2}\left(B_{1}\right)}+\|v\|_{L^{2}\left(\partial B_{1}\right)}\right)
$$

as desired.

Unlike in the one-space-dimensional case, we do not have an explicit upper bound for the maximal time of existence, and we are not able to provide a detailed description of the transition to nonexistence (as was done in [5]). We do suspect, based on numerical evidence (given the imposed symmetries on the initial data $f$ ) that blowup occurs at $n$ symmetrically distributed points on $\partial B_{1}$. Our two-dimensional analogue of Proposition 2.4 now reads as follows.

Proposition 3.8. Let $\phi_{n}$ denote the steady-state solution defined by (23). Let $u(x, y, t)$, $(x, y) \in B_{1}, 0 \leq t<T$, be a classical solution to the diffusion problem (26)-(27), with initial condition $f$ that satisfies the same symmetry properties (24)-(25) as $\phi_{n}$, and the compatibility condition $\frac{\partial f}{\partial \mathbf{n}}=\lambda F(f)=\lambda \sinh (f)$ on $\partial B_{1}$. If additionally $|f(x, y)| \geq$ $m\left|\phi_{n}(x, y)\right|,(x, y) \in B_{1}$, for some $m>1$, then there exists $T_{\max }<\infty$ (depending on $f$ ) such that $T<T_{\max }$. 
Proof. Green's formula asserts that

$$
\begin{aligned}
\int_{B_{1}} u \Delta u d x d y & =-\int_{B_{1}}|\nabla u|^{2} d x d y+\int_{\partial B_{1}} \frac{\partial u}{\partial n} u d \sigma \\
& =-\int_{B_{1}}|\nabla u|^{2} d x d y+\lambda \int_{\partial B_{1}} \sinh (u) u d \sigma
\end{aligned}
$$

at any time $t$, where $\Delta$ and $\nabla$ refer to the Laplacian and the gradient applied to $u$ in the spatial variables only. Therefore, for any $\epsilon>0$,

$$
\frac{1}{2 \epsilon}\|\Delta u\|_{L^{2}\left(B_{1}\right)}^{2}+\frac{\epsilon}{2}\|u\|_{L^{2}\left(B_{1}\right)}^{2} \geq-\int_{B_{1}}|\nabla u|^{2} d x d y+\lambda \int_{\partial B_{1}} \sinh (u) u d \sigma
$$

or

$$
-\|\Delta u\|_{L^{2}\left(B_{1}\right)}^{2} \leq 2 \epsilon \int_{B_{1}}|\nabla u|^{2} d x d y-2 \epsilon \lambda \int_{\partial B_{1}} \sinh (u) u d \sigma+\epsilon^{2}\|u\|_{L^{2}\left(B_{1}\right)}^{2},
$$

at any fixed time $t$. Insertion of the estimate from Lemma 3.7 and a simple rearrangement now gives

$$
-\left(1+C \epsilon^{2}\right)\|\Delta u\|_{L^{2}\left(B_{1}\right)}^{2} \leq 2 \epsilon \int_{B_{1}}|\nabla u|^{2} d x d y-2 \epsilon \lambda \int_{\partial B_{1}} \sinh (u) u d \sigma+C \epsilon^{2}\|u\|_{L^{2}\left(\partial B_{1}\right)}^{2},
$$

at any time $t$, where the constant $C$ is independent of $u, \epsilon$ and $t$. For $\epsilon=1 / \sqrt{C}$ this immediately leads to

$$
-\|\Delta u\|_{L^{2}\left(B_{1}\right)}^{2} \leq \frac{1}{\sqrt{C}} \int_{B_{1}}|\nabla u|^{2} d x d y-\frac{\lambda}{\sqrt{C}} \int_{\partial B_{1}} \sinh (u) u d \sigma+\frac{1}{2}\|u\|_{L^{2}\left(\partial B_{1}\right)}^{2},
$$

at any time $t$. Let $E(v)$ denote the energy

$$
E(v)=\frac{1}{2} \int_{B_{1}}|\nabla v|^{2} d x d y-\lambda \int_{\partial B_{1}}(\cosh (v)-1) d \sigma .
$$

A simple calculation yields

$$
\frac{d}{d t} E(u(\cdot, t))=-\int_{B_{1}}|\Delta u|^{2} d x d y,
$$

at any time $t$. Remember: $u=u(x, y, t)$ is the solution to the diffusion problem. Using (50) we thus obtain

$$
\begin{aligned}
\frac{d}{d t} E(u(\cdot, t)) \leq & \frac{1}{\sqrt{C}} \int_{B_{1}}|\nabla u|^{2} d x d y-\frac{\lambda}{\sqrt{C}} \int_{\partial B_{1}} \sinh (u) u d \sigma+\frac{1}{2}\|u\|_{L^{2}\left(\partial B_{1}\right)}^{2} \\
\leq & \frac{2}{\sqrt{C}} E(u(\cdot, t))+\frac{2 \lambda}{\sqrt{C}} \int_{\partial B_{1}}(\cosh (u)-1) d \sigma-\frac{\lambda}{\sqrt{C}} \int_{\partial B_{1}} \sinh (u) u d \sigma \\
& \quad+\frac{1}{2}\|u\|_{L^{2}\left(\partial B_{1}\right)}^{2} \\
= & \frac{2}{\sqrt{C}} E(u(\cdot, t))-\frac{2}{\sqrt{C}} \int_{\partial B_{1}}\left(\lambda+\frac{\lambda}{2} \sinh (u) u-\lambda \cosh (u)-\frac{\sqrt{C}}{4} u^{2}\right) d \sigma .
\end{aligned}
$$

Now consider the function

$$
g(z)=\lambda+\frac{\lambda}{2} \sinh (z) z-\lambda \cosh (z)-\frac{\sqrt{C}}{4} z^{2} .
$$


Clearly there exist positive constants $M, D$, and $d$ such that

$$
|g(z)| \leq D \text { for }|z|<M \text {, and } g(z) \geq d e^{|z|} \text { for } M \leq|z| .
$$

We therefore obtain

$$
\int_{\partial B_{1}} g(u) d \sigma \geq-D \int_{\partial B_{1} \cap\{|u| \leq M\}} 1 d \sigma+d \int_{\partial B_{1} \cap\{|u|>M\}} e^{|u|} d \sigma .
$$

Now suppose that the solution $u$ to the diffusion problem exists for all time. From the exponential lower bound of Proposition 3.6 it follows that

$$
\left|\phi_{n}(x, y)\right| \geq \delta \text { implies that }|u(x, y, t)| \geq c_{K} \delta e^{K t} \text { for all time } t,
$$

and thus

$$
\begin{aligned}
\partial B_{1} \cap\left\{(x, y):\left|\phi_{n}(x, y)\right| \geq \delta\right\} & \subseteq \partial B_{1} \cap\left\{(x, y):|u(x, y, t)| \geq c_{K} \delta e^{K t}\right\} \\
& \subseteq \partial B_{1} \cap\{(x, y):|u(x, y, t)| \geq M\},
\end{aligned}
$$

for $t>K^{-1} \log \left[M /\left(c_{K} \delta\right)\right]$. Now select $\delta>0$ strictly inside the range of $\phi_{n}$. The above inclusions, in connection with (52), yield

$$
\int_{\partial B_{1}} g(u(x, y, t)) d \sigma \geq-D \int_{\partial B_{1} \cap\{|u| \leq M\}} 1 d \sigma+d e^{c_{K} \delta e^{K t}} \int_{\partial B_{1} \cap\left\{\left|\phi_{n}(x, y)\right| \geq \delta\right\}} d \sigma .
$$

With

it follows that

$$
c_{\delta}=d \int_{\partial B_{1} \cap\left\{\left|\phi_{n}(x, y)\right| \geq \delta\right\}} d \sigma>0
$$

$$
-\int_{\partial B_{1}} g(u(x, y, t)) d \sigma \leq 2 \pi D-c_{\delta} e^{c_{K} \delta e^{K t}},
$$

for $t>K^{-1} \log \left[M /\left(c_{K} \delta\right)\right]$. Note that the constants $D$ and $c_{\delta}$ are positive and independent of $K$ (and $t$ ). Insertion of this into the inequality (51) gives

$$
\frac{d}{d t}\left(e^{-\frac{2 t}{\sqrt{C}}} E(u(\cdot, t))\right) \leq D_{1} e^{-\frac{2 t}{\sqrt{C}}}-d_{\delta} e^{C_{K} \delta e^{K t}-\frac{2 t}{\sqrt{C}}} \leq D_{1} e^{-\frac{2}{\sqrt{C}} t}-d_{K} e^{K t},
$$

for $t$ sufficiently large. Here $K>0$ is arbitrary, and the constants $C, D_{1}$ and $d_{K}$ are positive. The constants $C$ and $D_{1}$ are independent of $K$. Select a fixed $t_{*}$, sufficiently large. By integration of (54) from $t_{*}$ to $t$ we now get

$$
\begin{aligned}
E(u(\cdot, t)) \leq & e^{\frac{2\left(t-t_{*}\right)}{\sqrt{C}}} E\left(u\left(\cdot, t_{*}\right)\right)+D_{1} \frac{\sqrt{C}}{2}\left(e^{\frac{2\left(t-t_{*}\right)}{\sqrt{C}}}-1\right) \\
& -\frac{d_{K}}{K} e^{2 t / \sqrt{C}}\left(e^{K t}-e^{K t_{*}}\right) \text { for } t>t_{*} .
\end{aligned}
$$

From this formula it follows immediately that $E(u(\cdot, t))$ becomes negative for $t$ sufficiently large (recall that $K$ is arbitrary and $C$ is independent of $K$ ). However, in this case, the energy criterion of Proposition 4.1 in the appendix asserts that $u$ will cease to exist after a finite time, a clear contradiction to our supposition of global existence. We conclude that the classical solution $u$ must indeed cease to exist after a finite time, and this completes the proof of Proposition 3.8 . 
3.4. Example. To illustrate two-dimensional blowup in this setting, we show in Figure 3 the function $f(x, y)=1.01 \phi_{1}(x, y)$ with $\lambda=0.8$. We use $f$ as the initial condition $u(x, y, 0)=f(x, y)$ for the solution to (26)-(28); the maximum value for $f$ (at $(1,0)$ ) is about 0.479 . In Figure 4 we show the solution at time $t=1.34$; the maximum value is about 1.126. Past this time the solution appears to blow up (in the sense that our numerical solver no longer converges). In contrast, using the initial condition $f(x, y)=0.9 \phi_{1}(x, y)$, the solver yields a solution that rapidly converges to 0 (not shown).

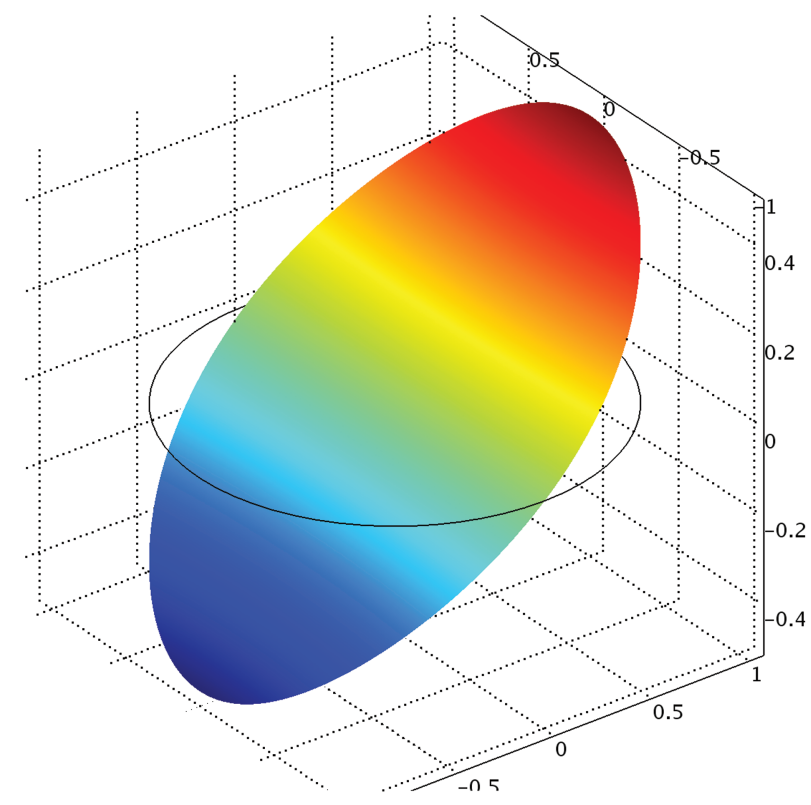

FIG. 3. Initial condition $f(x, y)=1.01 \phi_{1}(x, y)$ on unit disk.

4. Appendix: Concavity method for blowup. The argument presented in this appendix was originally adapted from the work of Quittner and Souplet (see e.g. [27]) by Kai Medville; it was done as part of Kai's Ph.D. work, although it did not actually appear in his dissertation [22. For completeness and the convenience of the reader we include the argument here.

In the context of this appendix, $\Omega$ can be any bounded domain in $\mathbb{R}^{N}$ for $N \geq 1$, with a suitably smooth boundary. Let $\lambda>0$ and $u \in C^{1}\left((0, T), C^{2}(\bar{\Omega})\right) \cap C^{0}\left([0, T), C^{1}(\bar{\Omega})\right)$ denote a solution to (26)-(28) with $f(x) \in C^{1}(\bar{\Omega})$, and $\partial f / \partial \mathbf{n}=\lambda \sinh (f)$ on $\partial \Omega$. It is well known that there exists such a solution $u(x, t)$ for some positive $T$. Let $T_{\max }$ denote the supremum of the $T$ 's for which a solution with these regularity properties exists.

Our goal is to show that, under a certain energy condition, $T_{\max }<\infty$. To this end, define the functional

$$
E(\phi):=\frac{1}{2} \int_{\Omega}|\nabla \phi|^{2} d x-\lambda \int_{\partial \Omega}(\cosh (\phi)-1) d \sigma_{x},
$$




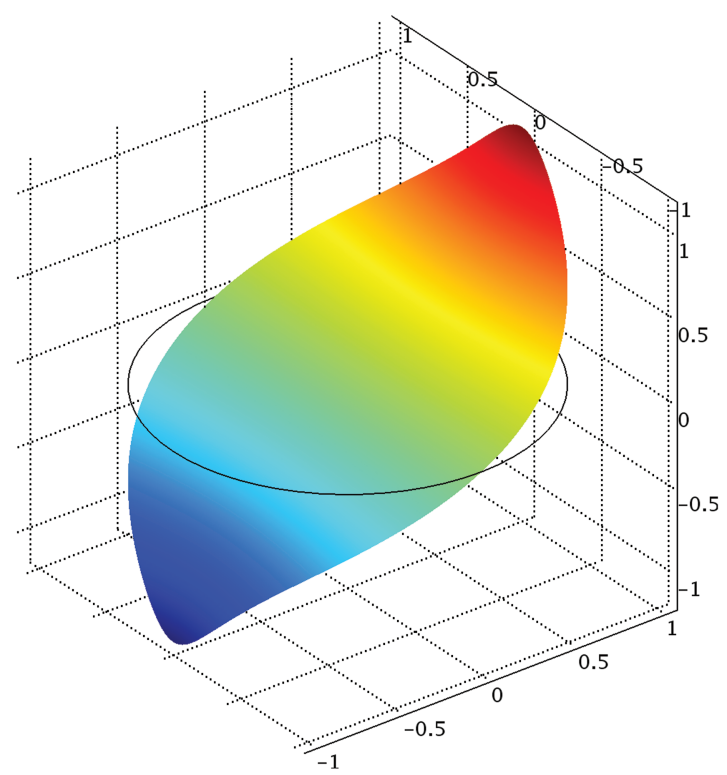

FIG. 4. Solution $u(x, y, 1.34)$ on unit disk.

where $\phi \in C^{1}(\Omega)$ (in fact, for $N \leq 2, E$ is well-defined on $H^{1}(\Omega)$ ). This is the energy functional associated with the steady-state problem

$$
\left\{\begin{aligned}
-\Delta v & =0 & & \text { in } \Omega \\
\frac{\partial v}{\partial \mathbf{n}} & =\lambda \sinh (v) & & \text { on } \partial \Omega .
\end{aligned}\right.
$$

Our main result is

Proposition 4.1. If the initial condition $f(x)$ satisfies $E(f)<0$, then $T_{\max }<\infty$.

More precisely, for $N \leq 2$, the argument we provide may be refined to show that the $H^{1}(\Omega)$ norm of the solution blows up in finite time.

Proof. The proof proceeds by contradiction, so let us assume that $T_{\max }=\infty$. For what follows, we introduce a second energy functional, namely the simple translation

$$
E_{D}(\phi):=\frac{1}{2} \int_{\Omega}|\nabla \phi|^{2} d x-\lambda \int_{\partial \Omega}(\cosh (\phi)-D) d \sigma_{x} .
$$

In particular, $E(\phi)=E_{1}(\phi)$ and $E_{D}(\phi)=E_{1}(\phi)+(D-1) \lambda|\partial \Omega|$. For any fixed $\phi$ the functional $E_{D}(\phi)$ is increasing in the parameter $D$. Note that strict negativity of the energy $E_{1}(f)$ means we can choose some $D_{0}>1$ such that $E_{D_{0}}(f)<0$. In fact, given $E_{D}(\phi)=E_{1}(\phi)+(D-1) \lambda|\partial \Omega|$, anything in the range

$$
1<D_{0}<1-\frac{E_{1}(f)}{\lambda|\partial \Omega|}
$$

will work.

Claim 4.2. If $u$ denotes the solution to (26)-(28), then for any $D$, the function $E_{D}(u(\cdot, t))$ is nonincreasing in $t$. 
The proof of this claim consists of a simple integration by parts. In the computation below the operators $\nabla$ and $\triangle$ are applied in the spatial variables only:

$$
\begin{aligned}
\frac{d}{d t} E_{D}(u(\cdot, t)) & =\int_{\Omega} \nabla u \cdot \nabla u_{t} d x-\lambda \int_{\partial \Omega} u_{t} \sinh (u) d \sigma_{x} \\
& =\int_{\Omega}\left(\nabla \cdot\left(u_{t} \nabla u\right)-u_{t} \Delta u\right) d x-\lambda \int_{\partial \Omega} u_{t} \sinh (u) d \sigma_{x} \\
& =\int_{\partial \Omega} u_{t} \frac{\partial u}{\partial \mathbf{n}} d \sigma_{x}-\int_{\Omega} u_{t} \Delta u d x-\lambda \int_{\partial \Omega} u_{t} \sinh (u) d \sigma_{x} \\
& =-\int_{\Omega}|\Delta u|^{2} d x \leq 0,
\end{aligned}
$$

where we have made use of the boundary condition (27) and $u_{t}=\Delta u$.

To continue with the proof of Proposition 4.1, define the functions

$$
g(t):=\frac{1}{2} \int_{\Omega} u(x, t)^{2} d x \text { and } G(t):=\int_{0}^{t} g(s) d s .
$$

Note that $G^{\prime}(t)=g(t)>0$ and $G(0)=0$.

Claim 4.3. The functions $g$ and $G$ satisfy

$$
\lim _{t \rightarrow \infty} g(t)=\infty \text { and } \lim _{t \rightarrow \infty} G(t)=\infty .
$$

The proof of this claim is a simple computation:

$$
\begin{aligned}
g^{\prime}(t) & =\int_{\Omega} u u_{t} d x=\int_{\Omega} u \Delta u d x \\
& =\int_{\Omega}\left(\nabla \cdot(u \nabla u)-|\nabla u|^{2}\right) d x \\
& =\int_{\partial \Omega} u \frac{\partial u}{\partial \mathbf{n}} d \sigma_{x}-\int_{\Omega}|\nabla u|^{2} d x \\
& =\lambda \int_{\partial \Omega} u \sinh (u) d \sigma_{x}-\int_{\Omega}|\nabla u|^{2} d x \\
& =-2 E_{1}(u(\cdot, t))+\lambda \int_{\partial \Omega}(u \sinh (u)-2 \cosh (u)+2) d \sigma_{x} \\
& \geq-2 E_{1}(u(\cdot, t)) \geq-2 E_{1}(f)>0,
\end{aligned}
$$

where we have used the fact that $x \sinh (x)-2 \cosh (x)+2 \geq 0$ for all $x \in \mathbb{R}$, as well as Claim 4.2. It follows that $\lim _{t \rightarrow \infty} g(t)=\infty$, and hence $\lim _{t \rightarrow \infty} G(t)=\infty$, which establishes Claim 4.3 .

Given our choice of $D_{0}>1$ above, let $\alpha=\alpha\left(D_{0}\right)>2$ be chosen so that the function

$$
h_{\alpha}(x):=x \sinh x-\alpha\left(\cosh x-D_{0}\right) \geq 0
$$

for all $x>0$. This is possible since for $D_{0}>1$ and all $x \in \mathbb{R}$ we have

$$
x \sinh x-2\left(\cosh x-D_{0}\right)>0,
$$

and since $x \sinh (x)$ grows faster than $\cosh (x)$ at infinity.

Claim 4.4. The function $G$ satisfies

$$
\left(G^{\prime}(t)-G^{\prime}(0)\right)^{2} \leq \frac{2}{\alpha} G(t) G^{\prime \prime}(t)
$$


To show this, we use the Cauchy-Schwarz inequality to find

$$
\begin{aligned}
\left(G^{\prime}(t)-G^{\prime}(0)\right)^{2} & =\left(\int_{0}^{t} G^{\prime \prime}(s) d s\right)^{2}=\left(\int_{0}^{t} \int_{\Omega} u u_{t} d x d s\right)^{2} \\
\leq & \left(\int_{0}^{t} \int_{\Omega} u^{2} d x d s\right)\left(\int_{0}^{t} \int_{\Omega}\left(u_{t}\right)^{2} d x d s\right)=2 G(t) \int_{0}^{t} \int_{\Omega}\left(u_{t}\right)^{2} d x d s
\end{aligned}
$$

From equation (56) we have

$$
\begin{aligned}
E_{D_{0}}(f)-E_{D_{0}}(u(t)) & =-\int_{0}^{t} E_{D_{0}}^{\prime}(s) d s \\
& =\int_{0}^{t} \int_{\Omega}(\Delta u)^{2} d x d s \\
& =\int_{0}^{t} \int_{\Omega} u_{t}(x, s)^{2} d x d s .
\end{aligned}
$$

We can combine inequalities (58) and (59) to find

$$
\left(G^{\prime}(t)-G^{\prime}(0)\right)^{2} \leq 2 G(t)\left(E_{D_{0}}(f)-E_{D_{0}}(u(t))\right) \leq-2 G(t) E_{D_{0}}(u(t)),
$$

since $E_{D_{0}}(f)<0$. With the above choice of $\alpha$ and $D_{0}$, we can rewrite $G^{\prime \prime}(t)$ as (refer to equation (57))

$$
\begin{aligned}
G^{\prime \prime}(t) & =g^{\prime}(t) \\
& =-\int_{\Omega}|\nabla u|^{2} d x+\lambda \int_{\partial \Omega} u \sinh (u) d \sigma_{x} \\
& =-\alpha E_{D_{0}}(u)+\left(\frac{\alpha-2}{2}\right) \int_{\Omega}|\nabla u|^{2} d x+\lambda \int_{\partial \Omega} h_{\alpha}(u) d \sigma_{x} \\
& \geq-\alpha E_{D_{0}}(u)
\end{aligned}
$$

since $\alpha>2$ and $h_{\alpha} \geq 0$. If we combine this with inequality (60) we obtain Claim 4.4,

To finish the proof of Proposition 4.1, we use the fact that $G^{\prime}(t)=g(t)$ limits to infinity to observe that, for any given $0<\epsilon<1$, there exists a $t_{*}$ such that

$$
1-\frac{G^{\prime}(0)}{G^{\prime}(t)} \geq 1-\frac{\epsilon}{2}>\sqrt{1-\epsilon} \text { for all } t \geq t_{*} .
$$

A little rearrangement yields

$$
\left(G^{\prime}(t)-G^{\prime}(0)\right)^{2}>(1-\epsilon)\left(G^{\prime}(t)\right)^{2} .
$$

In combination with Claim 4.4 this yields

$$
(1-\epsilon) \frac{\alpha}{2} G^{\prime}(t)^{2} \leq G(t) G^{\prime \prime}(t) .
$$

Since our choice of $\alpha>2$ has been made, we choose an $\epsilon>0$ small enough that $\beta=$ $(1-\epsilon) \frac{\alpha}{2}>1$. We then have the differential inequality

$$
\beta G^{\prime}(t)^{2} \leq G(t) G^{\prime \prime}(t)
$$

for some $\beta>1$ and all $t>t_{*}$. This inequality may be rewritten as

$$
(\beta-1)\left(G^{\prime}(t)\right)^{2} \leq G(t) G^{\prime \prime}(t)-\left(G^{\prime}(t)\right)^{2},
$$


which after division by $-\left(G^{\prime}\right)^{2}$ gives

$$
1-\beta \geq-\frac{G(t) G^{\prime \prime}(t)-\left(G^{\prime}(t)\right)^{2}}{\left(G^{\prime}(t)\right)^{2}}=\frac{d}{d t}\left(\frac{G(t)}{G^{\prime}(t)}\right)
$$

for $t>t_{*}$. We integrate both sides above from $t=t_{*}$ to $t=t$ to find

$$
(1-\beta)\left(t-t_{*}\right) \geq \frac{G(t)}{G^{\prime}(t)}-\frac{G\left(t_{*}\right)}{G^{\prime}\left(t_{*}\right)} .
$$

This last inequality immediately leads to

$$
\begin{aligned}
\frac{G(t)}{G^{\prime}(t)} & \leq(1-\beta)\left(t-t_{*}\right)+\frac{G\left(t_{*}\right)}{G^{\prime}\left(t_{*}\right)} \\
& =(1-\beta) t+c_{1} \text { for } t>t_{*},
\end{aligned}
$$

where $c_{1}=\frac{G\left(t_{*}\right)}{G^{\prime}\left(t_{*}\right)}+(\beta-1) t_{*}$. The left side above is always positive, while the right side (since $\beta>1$ ) becomes negative for $t$ sufficiently large, a contradiction. This shows that the solution $u(x, t)$ cannot exist for all time and thus completes the proof of Proposition 4.1.

Acknowledgments. M.S. Vogelius thanks O. Kavian for interesting discussions concerning energy based blow-up criteria. The research of M.S. Vogelius was partially supported by NSF grant DMS-0604999.

\section{REFERENCES}

[1] J.M. Arrieta and A. Rodriguez-Bernal, Localization on the boundary of blow-up for reactiondiffusion equations with Nonlinear Boundary Conditions, Comm. in Partial Diff. Equations, 29, 2004, pp. 1127-1148. MR2097578 (2005f:35159)

[2] C. Bandle and H. Brunner, Blowup in diffusion equations: A survey, Journal of Computational and Applied Mathematics, 97, 1998, pp. 3-22. MR1651764 (99g:35061)

[3] K. Bryan and M. Vogelius, A uniqueness result concerning the identification of a collection of cracks from finitely many electrostatic boundary measurements, SIAM J. Math. Anal., 23, 1992, pp. 950-958. MR 1166567 (93f:35238)

[4] K. Bryan and M.S. Vogelius, Singular solutions to a nonlinear elliptic boundary value problem originating from corrosion modeling, Quarterly of Applied Math, 60, 2002, pp. 675-694. MR.1939006 (2003i:35108)

[5] K. Bryan and M.S. Vogelius, Precise bounds for finite time blowup of solutions to very general one-space-dimensional nonlinear Neumann problems, to appear in the Quarterly of Applied Math.

[6] Y.P. Chen and C.H. Xie, Global existence and nonexistence for a strongly coupled parabolic system with nonlinear boundary conditions, Acta Mathematica Sinica, 22, 2006, pp. 1297-1304. MR.2251391 (2007c:35063)

[7] M. Chlebik and M. Fila, On the blow-up rate for the heat equation with a nonlinear boundary condition, Mathematical Methods in the Applied Sciences, 23, 2000, pp. 1323-1330. MR.1784465 (2001k:35176)

[8] R. Courant and D. Hilbert, Methods of Mathematical Physics, Vol. I, Wiley, New York, 1953. MR0065391 (16:426a)

[9] M. Fila and J. Filo, Blow-up on the boundary: A survey, pp. 67-78 in Singularities and Differential Equations, S. Janeczko et al. (eds), Banach Center Publ. 33, Polish Acad. Sciences, Warsaw, 1996. MR:1449147 (98c:35076)

[10] M. Fila, J. Filo and G.M. Lieberman, Blow-up on the boundary for the heat equation, Calculus of Variations and PDE, 10, 2000, pp. 85-99. MR.1803975 (2001k:35177)

[11] M. Fila and J. Guo, Complete blow-up and incomplete quenching for the heat equation with a nonlinear boundary condition, Nonlinear Analysis, 48, 2002, pp. 995-1002. MR1880259 
[12] M. Fila and P. Quittner, The blow-up rate for the heat equation with a nonlinear boundary condition, Mathematical Methods in the Applied Sciences, 14, 1991, pp. 197-205. MR.1099325 (92a:35023)

[13] Y. Giga and R. Kohn, Asymptotically self-similar blow-up of semilinear heat equations, Comm. Pure Appl. Math, 38, 1985, pp. 297-319. MR784476 (86k:35065)

[14] Y. Giga and R. Kohn, Characterizing blowup using similarity variables, Indiana Univ. Math. J., 36, 1987, pp. 425-447. MR.876989 (88c:35021)

[15] Y. Giga and R. Kohn, Nondegeneracy of blowup for semilinear heat equations, Comm. Pure Appl. Math 42, 1989, pp. 845-884. MR1003437|(90k:35034)

[16] J.S. Guo and B. Hu, Blowup rate for heat equation in Lipschitz domains with nonlinear heat source terms on the boundary, Journal of Mathematical Analysis and Applications, 269, 2002, pp. 28-49. MR 1907872 (2003c:35098)

[17] B. Hu and Z. Yin, The profile near blowup time for solution of the heat equations with a nonlinear boundary condition, Transactions Amer. Math. Soc., 346, 1994, pp. 117-135. MR1270664 (95c:35040)

[18] S. Kichenassamy, Recent Progress on Boundary Blow-up, pp. 329-341 in "Elliptic and Parabolic Problems", Volume 63 of the Book Series "Progress in Nonlinear Differential Equations and Their Applications", Birkhäuser, Basel, 2005. MR2176725 (2006e:35022)

[19] H.A. Levine: Some nonexistence and instability theorems for solutions of formally parabolic equations of the form $P u_{t}=-A u+F(u)$. Arch. Rational Mech. Anal., 51, 1973, pp. 371-386. MR 0348216 (50:714)

[20] H. Levine and L. Payne, Nonexistence theorems for the heat equation with nonlinear boundary conditions and for the porous medium equation backward in time, J. Differential Equations, 16, 1974, pp. 319-334. MR0470481(57:10235)

[21] Z. Lin and C. Xie, The blow-up rate for a system of heat equations with nonlinear boundary conditions, Nonlinear Analysis, 34, 1998, pp. 767-778. MR.1634742(2000a:35096)

[22] K. Medville, Existence and blow up behavior of planar harmonic functions satisfying certain nonlinear Neumann boundary conditions, Ph.D. dissertation, Department of Mathematics, Rutgers University (NJ), May 2005.

[23] M. Protter and H. Weinberger, Maximum Principles in Differential Equations, Springer, New York, 1999. MR762825 (86f:35034)

[24] F. Quiros and J.D. Rossi, Blow-up sets for linear diffusion equations in one dimension, Z. Angew. Math. Phys., 55, 2004, pp. 357-362. MR2047293 (2004m:35153)

[25] F. Quiros, J.D. Rossi and J.L. Vazquez, Complete blow-up and thermal avalanche for heat equations with nonlinear boundary conditions, Comm in PDE, 27, 2002, pp. 395-424. MR.1886965 (2002k:35041)

[26] P. Quittner and A. Rodriguez-Bernal, Complete and energy blow-up in parabolic problem with nonlinear boundary conditions, Nonlinear Analysis, 62, 2005, pp. 863-875. MR2153217 (2006a:35156)

[27] P. Quittner and P. Souplet, Superlinear Parabolic Problems: Blow-up, Global Existence and Steady States, Birkhaüser, Basel, 2007. MR.2346798 (2008f:35001)

[28] C. Roberts, Recent results on blow-up and quenching for nonlinear Volterra equations, J. Comput. Applied Math., 205, 2007, pp. 736-743. MR2329649 (2008c:45006)

[29] A. Rodriguez-Bernal and A. Tajdine, Nonlinear balance for reaction-diffusion equations under nonlinear boundary conditions: dissipativity and blow-up, J. Diff. Eqns., 169, 2001, pp. 332-372. MR $1808470(2002 \mathrm{c}: 35162)$

[30] J. Rossi, The blow-up rate for a semilinear parabolic equation with a nonlinear boundary condition, Acta Math. Univ. Comenianae, 2, 1998, pp. 343-350. MR1739446 (2000k:35133)

[31] W. Walter, On existence and nonexistence in the large of solutions of parabolic differential equations with a nonlinear boundary condition, SIAM J. Math. Anal., 6, 1975, pp. 85-90. MR0364868 $(51: 1122)$

[32] M. Wang and Y. Wu, Global existence and blow-up problems for quasilinear parabolic equations with nonlinear boundary conditions, SIAM J. Math. Anal., 24, 1993, pp. 1515-1521. MR1241155 (95c:35132)

[33] X.H. Yang, F.C. Li and C.H. Xie, Global existence and blow up of solutions for parabolic system involving cross-diffusions and nonlinear boundary conditions, Acta Mathematica Sinica, 21, 2005, pp. 923-928. MR 2156972 (2006i:35141)

[34] L. Zhao and S. Zheng, Blow-up estimates for system of heat equations coupled via nonlinear boundary flux, Nonlinear Analysis, 54, 2003, pp. 251-259. MR.1979732 (2004b:35151) 
[35] S. Zheng, F. Li, and B. Liu, Asymptotic behavior for a reaction-diffusion equation with inner absorbtion and boundary flux, Applied Math. Letters, 19, 2006, pp. 942-948. MR2240489 (2007b:35202) 